\title{
Nuclear Transparency to Intermediate-Energy Protons
}

\author{
James J. Kelly \\ Department of Physics, University of Maryland, College Park, MD 20742
}

\begin{abstract}
Nuclear transparency in the $\left(e, e^{\prime} p\right)$ reaction for $135 \leq T_{p} \leq 800 \mathrm{MeV}$ is investigated using the distorted wave approximation. Calculations using densitydependent effective interactions, both empirical and theoretical, are compared with phenomenological optical potentials. We find that nuclear transparency is well correlated with proton absorption and neutron total cross sections and that calculations using density-dependent effective interactions provide the best agreement with data. Nuclear transparency calculations are compared with recent electron scattering data for $Q^{2}<2(\mathrm{GeV} / \mathrm{c})^{2}$. For $T_{p} \lesssim 200 \mathrm{MeV}$ we find that there is considerable sensitivity to the choice of optical model and that the empirical effective interaction provides the best agreement with the data, but remains $5-10 \%$ low. For $T_{p} \gtrsim 300 \mathrm{MeV}$ we find that there is much less difference between these models, but that the calculations significantly underpredict transparency data and that the discrepancy increases with $A$. The differences between Glauber and optical model calculations are related to their respective definitions of the semi-inclusive cross section. By using a more inclusive summation over final states the Glauber model emphasizes nucleonnucleon inelasticity, whereas with a more restrictive summation the optical model emphasizes nucleon-nucleus inelasticity; experimental definitions of the semi-inclusive cross section lie between these extremes.
\end{abstract}

Typeset using REVTEX 


\section{INTRODUCTION}

The transparency of nuclear matter to the propagation of an intermediate-energy nucleon is of fundamental importance to the interpretation of many nuclear reactions. The elastic scattering of intermediate-energy nucleons, here defined by the range $100 \lesssim T_{p} \lesssim 800$ $\mathrm{MeV}$, is usually described using a complex optical potential whose real part produces refraction and whose imaginary part produces absorption of the flux contained within the elastic channel. That flux reappears, of course, in other channels which include inelastic scattering, knockout, and other reactions. Phenomenological optical potentials are obtained by fitting the parameters of some hypothetical function to elastic scattering data; sometimes absorption or total cross section data are included in the analysis also. Excellent fits to the data for a particular target and energy can usually be achieved, but the fitted parameters often vary erratically with respect to either mass or energy. Global analyses of more extensive data sets which impose smooth dependencies on energy and/or mass, usually at the expense of local fit quality, are expected to produce more realistic optical potentials. The optical potential is then presumed to represent the nucleon wave function within the medium with sufficient accuracy for the analysis of other reactions involving one or more nucleons of similar energy in either the initial or the final channel, such as $\left(e, e^{\prime} N\right)$ or $\left(p, p^{\prime} N\right)$.

However, since elastic scattering is determined by asymptotic phase shifts, any potential which produces the same set of phase shifts will predict the same elastic scattering even if the interior wave functions are quite different. Thus, various phase-equivalent optical potentials can produce significantly different predictions when employed to analyze other reactions. Alternatively, we have developed an empirical effective interaction (EEI) [1] 6] based upon the density dependence predicted by nuclear matter theories of the effective interaction, but where the parameters are fitted to inelastic scattering data. Transition densities fitted to electroexcitation data are used to minimize uncertainties due to nuclear structure. Both elastic and inelastic potentials are obtained by folding the same density-dependent effective interaction with transition densities using the local density approximation. Since both the distorted waves and the transition potentials depend upon the same interaction, the fitting procedure involves a self-consistency cycle which converges quite quickly. Several inelastic transitions, possibly among several targets, are fitted simultaneously, producing an empirical effective interaction (EEI) that depends upon local density but which is independent of target. Elastic scattering data can be analyzed also, but the interaction fitted to inelastic scattering data usually produces good elastic predictions whether or not elastic data are included.

Basing the phenomenology of the effective interaction primarily upon inelastic scattering offers several advantages over the determination of the optical potential via elastic scattering. First, the various radial shapes of transition densities provide differential sensitivity to the density dependence of the effective interaction, whereas the elastic optical potential requires only a global average over density. Second, since the distorted waves are determined self-consistently using optical potentials constructed from the same interaction that drives the inelastic transitions, the inelastic observables depend upon overlap integrals and are sensitive to the wave functions in the nuclear interior, thereby helping to determine the interior optical potential. Elastic scattering, on the other hand, depends on asymptotic

phase shifts and all potentials which share the same asymptotic wave functions predict the 
same elastic scattering despite differences in the interior. Therefore, provided that a consistent description of both elastic and inelastic scattering emerges from the phenomenological analysis of the effective interaction, the resultant optical potential should represent a more realistic description of the nuclear interior than models restricted to simple geometries, even if the latter yield better $\chi^{2}$ fits to the elastic scattering data alone. Finally, the EEI model requires far fewer parameters than traditional global optical models.

Single-nucleon knockout by electron scattering, $\left(e, e^{\prime} N\right)$ reactions, provide important tests for models of nucleon propagation. Since the nucleus is practically transparent with respect to the electron beam, the ejectile can originate from anywhere within the nuclear volume. Similarly, the smaller number of strongly absorbed wave functions gives $\left(e, e^{\prime} N\right)$ reactions better sensitivity to the interior than $(p, N)$ or $\left(p, p^{\prime} N\right)$ reactions. Hence, $\left(e, e^{\prime} p\right)$ reactions view the optical potential quite differently from $\left(p, p^{\prime}\right)$ reactions and there is no guarantee that the phenomenological potential that provides the best fit to proton elastic scattering data will also provide the best description of $\left(e, e^{\prime} p\right)$ data.

Quantitative analysis of missing-momentum distributions or spectroscopic factors for exclusive $A\left(e, e^{\prime} p\right) B$ reactions to discrete states of the residual nucleus depends upon accurate knowledge of absorption and distortion by final-state interactions (FSI) [0] 9 . Most experiments of that type have been performed using relatively low ejectile energies, typically $T_{p} \lesssim 135 \mathrm{MeV}$, where the EEI method is less successful. More recent experiments at Mainz, with $T_{p} \approx 200 \mathrm{MeV}$, and future experiments at CEBAF should be less sensitive to FSI uncertainties. Nevertheless, it is important to test those models of nuclear transparency directly.

The first measurements of nuclear transparency using electron scattering were made at MIT by Garino et al. [10,11] for $T_{p} \approx 180 \mathrm{MeV}$ by comparing inclusive cross sections with measurements of single-nucleon knockout cross sections that sampled the reaction cone and which were integrated with respect to missing energy. These semi-inclusive $\left(e, e^{\prime} p\right)$ data were analyzed using a correlated Glauber approximation by Pandharipande and Pieper [12], who found that Pauli blocking and short-range correlations are required to obtain transparencies large enough to reproduce the data. However, the Glauber model [13] is a high-energy approximation which postulates linear trajectories and hence is not expected to be particularly accurate for $T_{p} \approx 180 \mathrm{MeV}$. For proton elastic scattering the Glauber model is generally considered adequate for $T_{p} \gtrsim 800 \mathrm{MeV}$ [14,15]. Although integrated quantities, such as nuclear transparency, are probably less sensitive to the details of finalstate interactions such that the eikonal approximation may be sufficiently accurate at lower ejectile energies, the lower limit of the Glauber approximation to $\left(e, e^{\prime} p\right)$ has not yet been established.

At high $Q^{2}$, Brodsky [16] and Mueller [17] have predicted that nuclear transparency might be significantly enhanced by the phenomenon of color transparency $(\mathrm{CT})$, in which a Fock component of the nucleon wave function that is smaller in configuration space than the complete nucleon is ejected as an effectively color-neutral object that propagates with reduced interactions and increased attenuation length. Early calculations based upon the Glauber approximation with energy-independent nucleon-nucleon cross sections and various models of the hadron formation length for point-like configurations predicted substantial enhancement of nuclear transparency for the $A\left(e, e^{\prime} p\right)$ reaction at high $Q^{2}$ [18 21]. Nuclear transparency data using the $A\left(e, e^{\prime} p\right)$ reaction for $1.0 \leq Q^{2} \leq 6.8(\mathrm{GeV} / \mathrm{c})^{2}$ have recently 
been obtained at SLAC by the NE18 collaboration [22,23], but no definitive signal for CT was discerned 24. On the other hand, the data were limited by statistics and new data of much better accuracy are expected from CEBAF soon, although the maximum $Q^{2}$ will be smaller. However, more refined calculations, including the energy-dependence of the nucleon-nucleon interaction [15], finite range effects [25], Fermi motion [26,27], and coherency constraints [28], suggest that the onset of color transparency is rather slow and cannot be seen in these types of experiments unless $Q^{2} \gtrsim 30(\mathrm{GeV} / \mathrm{c})^{2}$. Hence, the CT signal in semi-inclusive $A\left(e, e^{\prime} p\right)$ cross sections is expected to be small at SLAC kinematics and even smaller at CEBAF kinematics. It has been suggested that for discrete states of the residual nucleus the attenuation coefficient for single nucleon knockout [19] or the asymmetry between missing momenta parallel versus antiparallel to the momentum transfer [29] might be more sensitive to color transparency at intermediate energies, but recent studies of the accuracy of the Glauber approximation suggest that $Q^{2} \gtrsim 2(\mathrm{GeV} / \mathrm{c})^{2}$ would be required to employ that model [30].

In this paper we report calculations of nuclear transparency for $135 \leq T_{p} \leq 800 \mathrm{MeV}$ using a distorted wave approximation (DWA). We compare calculations based upon the EEI model and a relativistic effective interaction, known as IA2 [31, 32], with a global optical potential from Dirac phenomenology [33]. For low energies we also consider several traditional nonrelativistic optical models. The calculations are compared with the MIT data 10,11 and with the low-energy data from SLAC experiment NE18 22,23. The model is presented in Sec. II. Predictions of proton absorption and neutron total cross sections using these optical potentials are compared with the available data for these closely related quantities in Sec. III. The DWA results for nuclear transparency are presented in Sec. IV. In Sec. $\mathrm{V}$ we compare our results with those obtained using the Glauber model. We find that the difference between these approaches arises from different definitions of the semi-inclusive cross section, with Glauber model being more inclusive and the optical model less inclusive than the experimental semi-inclusive cross section for intermediate-energy ejectiles. Finally, our conclusions are summarized in Sec. V1.

\section{MODEL}

\section{A. Definition of Nuclear Transparency}

The distorted spectral function is obtained experimentally by dividing the differential cross section by the off-shell electron-proton cross section, $\sigma_{e p}$, according to the ansatz

$$
\frac{d \sigma}{d \varepsilon_{f} d \Omega_{e} d \varepsilon_{p} d \Omega_{p}}=K \sigma_{e p} S^{D}\left(E_{m}, \mathbf{p}_{m}, \mathbf{p}^{\prime}\right)
$$

where $K$ is a kinematical factor. Final-state interactions between the ejectile and the residual nucleus make the distorted spectral function $S^{D}\left(E_{m}, \mathbf{p}_{m}, \mathbf{p}^{\prime}\right)$ depend upon the ejectile momentum $\mathbf{p}^{\prime}$, and on the angle between the initial and final nucleon momenta, whereas the (undistorted) spectral function would depend only on $E_{m}$ and $p_{m}$. Thus, the distorted spectral function depends upon the kinematical conditions and is different for parallel and 
perpendicular kinematics, for example. Furthermore, the dependence of $S^{D}$ upon the electron energy that arises from Coulomb distortion and from the properties of the electron current has been left implicit.

Due to the distribution of initial momenta, the quasifree single-nucleon knockout strength is spread over a Fermi cone whose opening angle is approximately $\theta_{F}=\tan ^{-1} k_{F} / q$ and over a range of missing energies that includes the binding energy for the deepest orbital. However, final-state interactions broaden these distributions and transfer some of the flux into more complicated final states. Hence, nuclear transparency can be loosely defined as the ratio between the coincident $\left(e, e^{\prime} p\right)$ cross section and the inclusive quasifree electron scattering cross section, where the concidence cross section is integrated over the Fermi cone and over the range of missing energy populated by direct knockout.

Optical models describe the loss of flux from the initial single-nucleon channels as absorption. Hence nuclear transparency is calculated in the optical model as the ratio

$$
\mathcal{T}_{w}=\frac{\int d E_{m} \int d^{3} p_{m} w\left(E_{m}, \mathbf{p}_{m}, \mathbf{p}^{\prime}\right) S^{D}\left(E_{m}, \mathbf{p}_{m}, \mathbf{p}^{\prime}\right)}{\int d E_{m} \int d^{3} p_{m} w\left(E_{m}, \mathbf{p}_{m}, \mathbf{p}^{\prime}\right) S^{P W}\left(E_{m}, p_{m}\right)}
$$

where in the numerator the distorted spectral distribution includes final-state interactions, and depends upon the ejectile momentum, whereas in the denominator the undistorted spectral distribution depends only upon the missing energy and momentum. The weight factor, $w\left(E_{m}, \mathbf{p}_{m}, \mathbf{p}^{\prime}\right)$, represents the experimental acceptance and distinguishes, for example, between parallel or quasiperpendicular kinematics; hence we distinguish various types of transparency functions using a subscript $w$ signifying the appropriate acceptance function. Clearly distortion and transparency depend upon the ejectile energy. However, since the distorted spectral function must be evaluated for the appropriate kinematical conditions, its dependence upon electron-scattering kinematics remains implicit in this definition. Therefore, to complete the definition of transparency we would need to specify the kinematical conditions of interest more completely, using the same integration regions and weighting factors for both numerator and denominator, where the appropriate weighting factors depend upon the kinematics of the experiment.

The simplest situation arises when $(\omega, q)$ are held constant, for which the nuclear transparency $\mathcal{T}_{\perp}$ may be defined as

$$
\mathcal{T}_{\perp}=\frac{\int d E_{m} \int d \theta \sin \theta S^{D}\left(E_{m}, p_{m}, p^{\prime}, \theta\right)}{\int d E_{m} \int d \theta \sin \theta S^{P W}\left(E_{m}, p_{m}\right)}
$$

where $\theta$ is the angle between the ejectile momentum $\mathbf{p}^{\prime}$ and the momentum transfer $\mathbf{q}$. Furthermore, it is simplest to require that the ejectile kinetic energy be constant in the barycentric frame so that final-state interactions can be evaluated for a unique ejectile energy. Also note that the weight factor should be expressed in terms of the center-of-mass system, where the ejectile momentum is constant, but for large $q$ the laboratory momentum changes little over the Fermi cone so that the distinction between the lab and $\mathrm{cm}$ angles matters little. However, for experiments using a narrow acceptance in $\omega$ with a large acceptance in ejectile kinetic energy $T_{p}$, it is necessary to employ energy-dependent optical potentials. Alternatively, for parallel kinematics we define 


$$
\mathcal{T}_{\|}=\frac{\int d E_{m} \int d p_{m} p_{m}^{2} S^{D}\left(E_{m}, p_{m}, p^{\prime}\right)}{\int d E_{m} \int d p_{m} p_{m}^{2} S^{P W}\left(E_{m}, p_{m}\right)}
$$

but must recognize that the ejectile momentum $p^{\prime}=p_{m}+q$ is correlated with $p_{m}$ and that the electron scattering kinematics again remain implicit. In this case it is also clearest to require the invariant mass for each $E_{m}$ to be constant despite the concomitant variation of electron kinematics.

We have verified that when the optical potentials are nullified, the distorted wave calculations result in unit transparency. However, it is important to recognize that these definitions of nuclear transparency can produce deviations from unity, with either sign, even when the optical potential is purely real because weighting functions based upon the plane-wave impulse approximation do not account for refractive effects. Although one might be tempted to divide out the refractive effect of the real potential by using a modified definition

$$
\tilde{\mathcal{T}}_{w}=\frac{\int d E_{m} \int d^{3} p_{m} w\left(E_{m}, \mathbf{p}_{m}, \mathbf{p}^{\prime}\right) S^{D}\left(E_{m}, \mathbf{p}_{m}, \mathbf{p}^{\prime}\right)}{\int d E_{m} \int d^{3} p_{m} w\left(E_{m}, \mathbf{p}_{m}, \mathbf{p}^{\prime}\right) S^{R}\left(E_{m}, \mathbf{p}_{m}, \mathbf{p}^{\prime}\right)}
$$

where $S^{R}\left(E_{m}, \mathbf{p}_{m}, \mathbf{p}^{\prime}\right)$ is the distorted spectral function for the real part of optical potential, our original definition, Eq. (2), conforms more closely to the customary experimental definition. The difference between these approaches is generally greatest when the summation over missing energy is restricted to a single state, or subshell. For quasiperpendicular kinematics, the refractive effects are minimized when measurements are made on both sides of $\mathbf{q}$ for closed shell targets so that the opposing effects of spin-orbit distortion are approximately balanced for both sides of $\mathbf{q}$ and for both spin-orbit partners. For parallel kinematics a net attractive (repulsive) real central potential shifts the missing momentum distributions to smaller (larger) $p_{m}$ and enhances (reduces) the peak values of $S^{D}\left(E_{m}, p_{m}\right)$ for positive (negative) missing momenta, but the net effect on transparency tends to balance when the integration over $p_{m}$ is symmetric. Since the shifts in the peak positions are also sensitive to spin-orbit distortion, the attenuation factors for spin-orbit partners can be different. Nevertheless, numerical studies show that for calculations using symmetric ranges of missing momenta the ratio $\mathcal{T}_{w} / \tilde{\mathcal{T}}_{w}$ remains within a few percent of unity for both parallel and quasiperpendicular kinematics for $A \geq 12$ and $135 \leq T_{p} \leq 800 \mathrm{MeV}$. Alternatively, one can assess the effects of refraction by comparing transparency calculations with and without the real parts of the optical potential. Thus, over the ranges of $A$ and $T_{p}$ considered, we find that $|\delta \mathcal{T}| / \mathcal{T} \lesssim 7 \%$ when the real parts of the optical potential are eliminated and conclude that the present definition does in fact provide an unambiguous measure of transparency that is rather insensitive to refraction. Therefore, we prefer to employ Eq. (2), despite its slight ambiguity between refraction and absorption, because it is closer to the experimental quantity of interest and the refractive effects do not significantly affect its interpretation in terms of absorption. Furthermore, we also find that

$$
\left|\frac{\mathcal{T}_{\|}-\mathcal{T}_{\perp}}{\mathcal{T}_{\perp}}\right| \lesssim 6 \%
$$

for $A \geq 12$ and $135 \leq T_{p} \leq 800 \mathrm{MeV}$; hence, we expect our calculations to be quite insensitive to the small range of missing momentum parallel to $\mathbf{q}$ that is inevitably accepted 
when performing measurements of $\mathcal{T}_{\perp}$. Therefore, we compare calculations of $\mathcal{T}_{\perp}$ directly to the experimental data without attempting to simulate the complete experimental acceptance functions.

\section{B. Spectral Function}

In the independent-particle model (IPM) the spectral functions take the forms

$$
\begin{gathered}
S_{I P M}\left(E_{m}, p_{m}\right)=\sum_{\alpha} S_{\alpha} \varrho_{\alpha}\left(p_{m}\right) \delta\left(E_{m}-E_{\alpha}\right) \\
S_{I P M}^{D}\left(E_{m}, \mathbf{p}_{m}, \mathbf{p}^{\prime}\right)=\sum_{\alpha} S_{\alpha} \varrho_{\alpha}^{D}\left(\mathbf{p}_{m}, \mathbf{p}^{\prime}\right) \delta\left(E_{m}-E_{\alpha}\right)
\end{gathered}
$$

where $E_{\alpha}$ and $S_{\alpha}$ are the missing energy and spectroscopic factor for orbital $\alpha$,

$$
\varrho_{\alpha}\left(p_{m}\right)=\left|\int d^{3} r e^{-i \mathbf{p}_{\mathbf{m}} \cdot \mathbf{r}} \phi_{\alpha}(\mathbf{r})\right|^{2}
$$

is the momentum distribution arising from a single-particle wave function $\phi_{\alpha}(\mathbf{r})$ normalized such that

$$
4 \pi \int d p_{m} p_{m}^{2} \varrho_{\alpha}\left(p_{m}\right)=1
$$

and $\varrho_{\alpha}^{D}\left(\mathbf{p}_{m}, \mathbf{p}^{\prime}\right)$ is the corresponding distorted momentum distribution. Hence the nuclear transparency for quasiperpendicular kinematics reduces to

$$
\mathcal{T}_{\perp}=\frac{\sum_{\alpha} S_{\alpha} \int d \theta \sin \theta \varrho_{\alpha}^{D}\left(p_{m}, p^{\prime}, \theta\right)}{\sum_{\alpha} S_{\alpha} \int d \theta \sin \theta \varrho_{\alpha}\left(p_{m}\right)} .
$$

Although interactions fragment the low-lying valence hole strength and populate the continuum for large missing energy and momentum, nuclear transparency is much more sensitive to final state interactions than to details of the spectral distribution. For a given orbital, attenuation depends more strongly on ejectile energy than on missing energy, so that the spreading of the hole strength is not expected to appreciably affect its contribution to the integrated yield, especially for experiments which select a relatively narrow range of ejectile energy. Similarly, although variations of the bound-state wave function which change the rms radius affect the missing momentum distribution, such variations have little effect on the integrated yield and tend to cancel in the ratio used for transparency. Therefore, for transparency calculations it is sufficient to employ IPM spectral functions, although correlations must be included to describe the distorted spectral distribution in detail.

For $A \leq 16$ we used Woods-Saxon single-particle wave functions, whereas for heavier nuclei bound-state wave functions were obtained using the Skyrme-Hartree-Fock model based upon the interaction designated $Z_{\sigma}$ [34, 35]. The single-particle energy spectra for each target were shifted to obtain the correct separation energies. For each orbital the final-state interactions were evaluated using ejectile energies based upon $\omega$ and the shifted HartreeFock separation energies. Note that the results are rather insensitive to the details of the bound-state wave functions, but are quite sensitive to the choice of optical model. 


\section{Distorted Wave Approximation}

The distorted wave approximation (DWA) for the electromagnetic transition amplitude that governs the single-nucleon knockout reaction $A\left(e, e^{\prime} N\right) B$ can be expressed in the form 36]

$$
\mathcal{M}=\int \frac{d^{3} q^{\prime}}{(2 \pi)^{3}} \mathcal{J}_{\mu}^{e}\left(\mathbf{q}^{\prime}\right) \frac{1}{Q^{\prime 2}} \mathcal{J}_{N}^{\mu}\left(\mathbf{q}^{\prime}\right)
$$

where the electron and nuclear currents are

$$
\begin{aligned}
& \mathcal{J}_{\mu}^{e}\left(\mathbf{q}^{\prime}\right)=\int d^{3} r e^{-i \mathbf{q}^{\prime} \cdot \mathbf{r}} \bar{\psi}_{f}^{e}(\mathbf{r}) \gamma_{\mu} \psi_{i}^{e}(\mathbf{r}) \\
& \mathcal{J}_{\mu}^{N}\left(\mathbf{q}^{\prime}\right)=\int d^{3} r e^{i \mathbf{q}^{\prime} \cdot \mathbf{r}} \bar{\psi}_{f}^{N}(\mathbf{r}) \Gamma_{\mu} \psi_{i}^{N}(\mathbf{r})
\end{aligned}
$$

and where $\Gamma_{\mu}$ is the vertex operator for the nucleon current. In these expressions the electron wave functions relative to the target of mass $m_{A}$ are denoted by the spinors $\psi_{i}^{e}$ and $\psi_{f}^{e}$ for the initial and final states, respectively. At this stage we leave implicit the dependence of the nuclear current upon the ejectile kinematics and the state of the residual nucleus. Since it is more convenient to express the ejectile wave functions $\psi^{N}$ relative to the residual nucleus of mass $m_{B}$, the radial scale is adjusted by means of the reduced momentum transfer [37] $\tilde{\mathbf{q}}^{\prime}=\mathbf{q}^{\prime} m_{B} / m_{A}$.

If we assume that a virtual photon with momentum $\mathbf{q}^{\prime}$ is absorbed by a single nucleon with initial momentum $\mathbf{p}$, the nuclear current at position $\mathbf{r}$ becomes

$$
\mathcal{J}_{\mu}^{N}(\mathbf{r})=\int \frac{d^{3} p}{(2 \pi)^{3}} \frac{d^{3} p^{\prime \prime}}{(2 \pi)^{3}} e^{-i \tilde{\mathbf{q}}^{\prime} \cdot \mathbf{r}} \tilde{\chi}^{(-) *}\left(\mathbf{p}^{\prime}, \mathbf{p}^{\prime \prime}\right) \Gamma_{\mu}\left(\mathbf{p}^{\prime \prime}, \mathbf{p}\right) \tilde{\phi}(\mathbf{p})
$$

where the single-nucleon wave function is the amplitude for removing a nucleon from the initial state of target $\mathrm{A}$ and reaching the final state of residual nucleus $\mathrm{B}$, such that

$$
\tilde{\phi}(\mathbf{p})=\langle B|a(\mathbf{p})| A\rangle .
$$

The distorted wave $\tilde{\chi}^{(-) *}\left(\mathbf{p}^{\prime}, \mathbf{p}^{\prime \prime}\right)$ is the amplitude that the ejectile with initial momentum

$\mathbf{p}^{\prime \prime}=\mathbf{p}+\mathbf{q}^{\prime}$ emerges from the nuclear field with final momentum $\mathbf{p}^{\prime}$. In coordinate space these wave functions are expressed as

$$
\begin{aligned}
\phi(\mathbf{r}) & =\int \frac{d^{3} p}{(2 \pi)^{3}} e^{i \mathbf{p} \cdot \mathbf{r}} \tilde{\phi}(\mathbf{p}) \\
\chi\left(\mathbf{p}^{\prime}, \mathbf{r}\right) & =\int \frac{d^{3} p^{\prime \prime}}{(2 \pi)^{3}} e^{i \mathbf{p}^{\prime \prime} \cdot \mathbf{r}} \tilde{\chi}\left(\mathbf{p}^{\prime}, \mathbf{p}^{\prime \prime}\right) .
\end{aligned}
$$

Thus, the nuclear current becomes

$$
\mathcal{J}_{\mu}^{N}\left(\mathbf{p}^{\prime}, \mathbf{q}^{\prime}\right)=\int \frac{d^{3} p}{(2 \pi)^{3}} \tilde{\chi}^{(-) *}\left(\mathbf{p}^{\prime}, \mathbf{p}+\mathbf{q}^{\prime}\right) \Gamma_{\mu}\left(\mathbf{p}+\mathbf{q}^{\prime}, \mathbf{p}\right) \tilde{\phi}(\mathbf{p})
$$

where $\mathbf{q}^{\prime}$ is the local momentum transfer supplied by the electron. 
Since nuclear transparency depends upon the nuclear final-state interactions and not the electronuclear initial-state interactions, distortion of the electron wave function should either be included in both numerator and denominator of Eq. (2) or excluded from both. To a good approximation, Coulomb distortion can be described as a shift of the effective momentum transfer and a focussing factor which increases the virtual-photon flux [38,39]. Since these aspects of Coulomb distortion have similar effects upon both exclusive and inclusive electron scattering, it is reasonable to omit Coulomb distortion for both. In the absence of Coulomb distortion, the electron current is proportional to $\delta^{3}\left(\mathbf{q}^{\prime}-\mathbf{q}\right)$, so that the nuclear current can be evaluated for a unique value of the momentum transfer obtained from asymptotic kinematics. Therefore, we obtain

$$
\mathcal{J}_{\mu}^{N}\left(\mathbf{p}^{\prime}, \mathbf{q}\right) \approx \int d^{3} r e^{i \tilde{\mathbf{q}} \cdot \mathbf{r}} \chi^{(-) *}\left(\mathbf{p}^{\prime}, \mathbf{r}\right) \Gamma_{\mu}\left(\mathbf{p}^{\prime}, \mathbf{p}^{\prime}-\mathbf{q}\right) \phi(\mathbf{r})
$$

where the vertex function has now been reduced to a matrix, acting on nucleon spins, whose elements are evaluated using effective kinematics.

We used the $c c 1$ vertex function of de Forest [40] with nucleon form factors from model 3 of Gari and Krümpelmann [41,42]. Current conservation was enforced at the one-body level by eliminating the longitudinal in favor of the charge component. However, it is important to note that the transparency calculations are quite insensitive to these choices for the vertex function and, in fact, are quite insensitive to the electron-scattering kinematics also. The most important variables are the ejectile energy and the choice of optical model.

\section{Optical Models}

Distorted waves were obtained from solutions to a Schrödinger equation of the form

$$
\left(\nabla^{2}+k_{\alpha}^{2}-2 \mu_{\alpha} U_{\alpha}\right) \xi_{\alpha}\left(\boldsymbol{k}_{\alpha}, \boldsymbol{r}\right)=0
$$

where relativistic kinematics are incorporated by interpreting $k_{\alpha}$ as the exact relativistic wave number and $\mu_{\alpha}$ as the reduced energy for channel $\alpha$ [43]. We assume that the optical potential can be reduced to local form and that nonspherical components may be neglected. Thus, the optical potential takes the form

$$
U_{\alpha}(r)=U_{\alpha}^{Z}(r)+U_{\alpha}^{C}(r)+U_{\alpha}^{L S}(r) \mathbf{L} \cdot \boldsymbol{\sigma}
$$

where $U_{\alpha}^{Z}$ is the Coulomb potential, $U_{\alpha}^{C}$ is the central potential, and $U_{\alpha}^{L S}=\frac{1}{r} \frac{\partial F^{L S}}{\partial r}$ is the spin-orbit potential for exit channel $\alpha$. Although in principle the optical potential for each exit channel depends upon the structure of the residual nucleus, we employ a mean field approximation for single-nucleon knockout in which there is only a small kinematic dependence upon the ejectile energy. To accomodate models which include nonlocality corrections, we identify the distorted wave function as

$$
\chi_{\alpha}\left(\boldsymbol{k}_{\alpha}, \boldsymbol{r}\right)=P(r) \xi_{\alpha}\left(\boldsymbol{k}_{\alpha}, \boldsymbol{r}\right)
$$

where $P(r)$ is a Perey factor which is unity for local models, approaches unity at large distances for nonlocal models, and which may be complex. 


\section{Nonrelativistic Woods-Saxon Potentials}

The potential fitted by Schwandt et al. 444 to cross section and analyzing power for proton elastic scattering for $A \geq 40$ and $80 \leq T_{p} \leq 180 \mathrm{MeV}$ is commonly employed for knockout analyses. It may be used for either proton or neutron scattering since it contains a parametrization of the symmetry potential. Although the Schwandt potential does not bear extrapolation in either mass or energy well, it is nevertheless often used for lighter nuclei, sometimes even for mass-12. A potential developed by Abdul-Jalil and Jackson [45,46] for $A \approx 12$ and $50 \leq T_{p} \leq 160 \mathrm{MeV}$ has sometimes been used for knockout studies, but in our opinion its description of proton scattering data is unsatisfactory. Alternatively, the $p+{ }^{12} \mathrm{C}$ potential of Comfort and Karp [47] for $T_{p} \leq 185 \mathrm{MeV}$ is preferred. Unfortunately, a global nonrelativistic optical potential for $A \geq 12$ and a broad range of energy does not appear to exist. For that we must appeal to Dirac phenomenology (DP).

Many analyses of single-nucleon knockout also include a Perey nonlocality correction of the form 48,49

$$
P(r)=\left[1-\frac{m_{p}}{2 \hbar^{2}} \beta^{2} V^{C}(r)\right]^{-1 / 2}
$$

where the central potential is separated into real and imaginary parts denoted $U^{C}(r)=$ $V^{C}(r)+i W^{C}(r)$. The nonlocality parameter is typically chosen as $\beta=0.85 \mathrm{fm}$, based upon the original analysis of neutron scattering for $T_{n} \leq 20 \mathrm{MeV}$. Although the applicability of this simple prescription has not been established for $T_{p}>100 \mathrm{MeV}$, when using nonrelativistic Woods-Saxon potentials, such as the Schwandt or the Comfort and Karp models, we conform to standard practice by including the Perey factor. Since the Perey factor is less than unity in the interior and equal to unity outside the potential, its effect is to reduce the transparency, especially for interior orbitals.

\section{Dirac Phenomenology}

Suppose that a four-component Dirac spinor,

$$
\Psi(\mathbf{r})=\left(\begin{array}{l}
\psi_{+}(\mathbf{r}) \\
\psi_{-}(\mathbf{r})
\end{array}\right)
$$

where $\psi_{+}$and $\psi_{-}$are two-component Pauli spinors for positive and negative energy components, satisfies a Dirac equation of the form

$$
[\boldsymbol{\alpha} \cdot \boldsymbol{p}+\beta(m+S)] \Psi=\left(E-V-V^{Z}\right) \Psi
$$

with scalar and vector potentials $S$ and $V$. Upon elimination of the lower components, an equivalent Schrödinger equation of the form

$$
\left[\nabla^{2}+k^{2}-2 \mu\left(U^{Z}+U^{C}+U^{L S} \boldsymbol{L} \cdot \boldsymbol{\sigma}\right)\right] \phi=0
$$

can be obtained, where $\psi_{+}$is related to $\phi$ by the Darwin transformation 


$$
\begin{aligned}
\psi_{+} & =B^{1 / 2} \phi \\
B & =1+\frac{S-V-V^{Z}}{E+m} .
\end{aligned}
$$

The Schrödinger solutions are phase-equivalent to the Dirac solutions in the sense that the asymptotic phase shifts, and hence observables for elastic scattering, are the same. However, the Dirac wave function is modified in the interior by a nonlocality factor similar in form to the Perey-Buck nonlocality factor, except that it depends upon $S-V$, which is closely related to the spin-orbit potential, rather than upon the central potential. Thus, $B$ can be deduced directly from the spin-orbit potential [50]. When used in nonrelativistic calculations, the positive energy spinor $\psi_{+}$, including the Darwin factor, is identified with the distorted wave $\chi$.

Hama et al. [51] produced global Dirac optical potentials for $A \geq 40$ and $65 \leq T_{p} \leq 1040$ $\mathrm{MeV}$. The global Dirac optical potential was then extended by Cooper et al. [33] to the ranges $A \geq 12$ and $20 \leq T_{p} \leq 1040 \mathrm{MeV}$. Of the several essentially equivalent variations of the global potential that were provided, we have chosen the version labelled EDAD1. Although the scalar+vector (SV) model of Dirac phenomenolgy is not unique, and relatively simple but arbitrary shapes are employed for the potentials, this work represents the most extensive and systematic analyses of proton optical potentials available. The available proton-nucleus elastic scattering data are described very well by potentials whose properties vary smoothly with both mass and energy.

\section{E. Local Density Approximation}

Optical potentials can also be obtained by folding the nucleon-nucleon effective interaction with the nuclear density distribution. In recent years it has become clear that the intermediate-energy nucleon-nucleon effective interaction depends strongly upon the density in the interaction region. Several calculations of the effective interaction in nuclear matter have been made following the seminal work of Hüfner and Mahaux [52]. Jeukenne, Lejeune, and Mahaux [53 56] computed the self-energy and the optical potential for $0 \leq T_{p} \leq 160$ $\mathrm{MeV}$ using the Reid soft-core potential [57]. Brieva, Rook, and von Geramb [58 62] developed a Brueckner-Hartree-Fock (BHF) approach and used the Hamada-Johnston potential [63] to calculate the pair wave function in nuclear matter, from which a local pseudopotential was constructed for $T_{p} \leq 180 \mathrm{MeV}$ using a generalization of the Siemens averaging procedure [64]. Similar calculations using the Hamada-Johnston potential have also been performed by Yamaguchi, Nagata, and Michiyama (YNM) [65,66], who parametrized their results for $T_{p} \leq 200 \mathrm{MeV}$ in Gaussian rather than Yukawa form. The BHF approach was refined by von Geramb and collaborators [67,68], who constructed an effective interaction based upon the Paris potential [69], designated Paris-Hamburg (PH), that is applicable for $100 \leq T_{p} \leq 400 \mathrm{MeV}$. Nakayama and Love [70] used the Bonn potential [71] to calculate a local pseudopotential that reproduces on-shell matrix elements of the G-matrix. These theories are all based upon the Bethe-Goldstone equation and include Pauli blocking and self-energy corrections self-consistently. A closely related calculation by Ray [72 used a coupled channels nucleon-isobar model and Watson multiple scattering theory to calculate 
a density-dependent t-matrix applicable to nucleon energies above $200 \mathrm{MeV}$ that includes some of the effects of pion production. Finally, Furnstahl, Wallace, and Kelly [31,32 have developed an effective interaction in a form similar to the EEI model based upon the relativistic IA2 model. Density dependence arises from the distortion of Dirac spinors in the nuclear medium, which primarily affects the real central interaction, and Pauli blocking, which damps the absorptive potential. The model naturally provides stronger density dependence for inelastic scattering than for elastic scattering, which is needed to describe the data.

All of these calculations predict strong density dependence of the nucleon-nucleon effective interaction. The dominant effect for the real central interaction is equivalent to a short-ranged repulsive interaction that is proportional to density and nearly independent of energy. In the BHF approaches this short-range repulsive interaction arises both from the anticorrelation between identical nucleons in nuclear matter and from dispersive effects in the self-consistent mean field, whereas in the IA2 approach it arises from spinor distortion in the strong scalar and vector mean fields. However, both approaches predict that the density dependence of the real central interaction depends slowly upon energy and remains quite strong even at $800 \mathrm{MeV}$ 43,32]. The dominant effect for the imaginary central interaction arises from Pauli blocking and for both the BHF and the IA2 models gives results similar to the familiar Clementel-Villi [73] damping of the absorptive potential, in which the damping factor is inversely proportional to the proton energy. Hence this effect is most important for low energies.

Although the qualitative features of the medium modifications are essentially the same, the quantitative differences among the various theories are surprisingly large, much larger than would be expected from the variations among the underlying nucleon-nucleon potentials that are employed, suggesting that the approximations required to evaluate the effective interaction are not yet under good control. The effect of these differences upon elastic and inelastic scattering calculations has been surveyed in a series of papers by Kelly and collaborators in which transition densities measured by electroexcitation are used to minimize uncertainties due to nuclear structure and to isolate the effective interaction for detailed examination [1 [6]. Transition densities which are strong in the interior provide information about the high-density properties of the effective interaction, whereas surface-peaked transition densities reveal the low-density properties. The systematic comparison of such cases demonstrates quite clearly that the effective interaction depends upon local density and that estimates based upon nuclear matter theory have qualitatively correct characteristics, but that none of the theories presently available is sufficiently accurate for quantitative applications to nuclear structure. Therefore, an empirical model of the effective interaction was developed in which medium modifications similar to those predicted by nuclear matter theory are parametrized in a form suitable to phenomenological analysis of data. The parameters are adjusted to reproduce inelastic scattering data for several states in one or more targets simultaneously. Empirical effective interactions have been extracted from data for several energies in the range $100 \leq T_{p} \leq 650 \mathrm{MeV}$. For each energy we find that a unique effective interaction describes data for several inelastic transitions in a single nucleus and that the fitted interaction is essentially independent of target. These findings tend to confirm the basic hypothesis of the local density approximation (LDA), namely that the interaction depends primarily upon local density and is independent of the detailed structure of any 
particular target or transition. The fitted parameters also exhibit a relatively smooth energy dependence.

Although slightly better fits to some of the data sets may be found in the original analyses, for the present purposes we choose to employ the empirical effective interactions (EEI) tabulated by Kelly and Wallace [32]. For this set of interactions, the medium modifications are applied to the Franey-Love (FL) [74] parametrization of the free $t$-matrix, which is available for all relevant energies, and common fitting strategies and constraints were used to help smooth the energy dependence of the fitted parameters. These choices are made primarily for aesthetic reasons and have very little effect upon knockout calculations.

In the local density approximation (LDA), the central and spin-orbit potentials become

$$
\begin{aligned}
U^{C}(r) & =\frac{2}{\pi} \int d q q^{2} j_{0}(q r) t^{C}(q, \rho) \tilde{\rho}(q) \\
F^{L S}(r) & =\frac{2}{\pi} \int d q q^{2} j_{0}(q r) t^{\prime L S}(q, \rho) \tilde{\rho}(q)
\end{aligned}
$$

where

$$
\tilde{\rho}(q)=\int d r r^{2} j_{0}(q r) \rho(r)
$$

is the Fourier transform of the ground-state density $\rho$. Note that a sum over nucleon or isospin indices has been left implicit. To minimize uncertainties due to the nuclear density, the proton density was obtained by unfolding the proton charge form factor from the charge densities measured by electron scattering and tabulated in Refs. [75]. For relatively small momentum transfers, charge symmetry ensures that the neutron and proton densities for mirror nuclei are very nearly proportional to each other and since the high $q$ properties of distorting potentials have very little effect upon knockout calculations, especially for integrated strengths, we use $\rho_{n}(r)=N \rho_{p}(r) / Z$. Furthermore, we evaluate the local density at the site of the projectile.

Since the empirical effective interaction was fitted to data for elastic and inelastic scattering self-consistently without explicit use of a nonlocality correction, no Perey factor is used with the EEI model for knockout. Similarly, the IA2 interaction is local by construction and does not require a Perey factor either. Some other versions of the LDA do involve nonlocality corrections based upon the exchange contribution or upon the momentum dependence of the effective mass, but are not employed here because none of those models provide adequate descriptions of the proton scattering data.

The potentials which emerge from all microscopic models exhibit much more complicated radial shapes than posited by the Woods-Saxon model of the optical potential. The detailed shapes depend upon the density dependence and range of the effective interaction and upon the nuclear density, which especially for light targets is not well approximated by the Fermi shape. For energies between about 100 and $300 \mathrm{MeV}$, for example, the real central potential exhibits a characteristic "wine-bottle" shape. Similar shapes also arise from the nonrelativistic reduction of either Dirac phenomenolgy or the relativistic impulse approximation. Although good fits to elastic scattering data may be achieved with simplistic models of the potential, artificially simple geometries cannot be justified on more fundamental grounds. Furthermore, the missing momentum distributions for discrete states do show some sensitivity to the shape of the real central potential. 


\section{COMPARISON OF OPTICAL MODELS}

Integrated quantities, such as nuclear transparency, are much more sensitive to attenuation of the flux than to distortion of the angular distribution by final state interactions. Therefore, in this section we compare data for proton absorption and neutron total cross sections with calculations based upon a variety of optical models for $100<T_{p}<800 \mathrm{MeV}$. More detailed analyses of proton elastic and inelastic scattering data can be found in Refs. [1] for example.

Predicted proton absorption cross sections are compared with data in Fig. 1. Unfortunately, the available proton data are scarce and of uneven quality [76]. Nevertheless, the EEI model provides accurate predictions for these data, although the $200 \mathrm{MeV}$ interaction appears to give results which are systematically low compared to the trends for other energies. It is important to remember that the EEI model is dominated by inelastic data, but gives good fits to elastic data whether or not they are included in the analysis [77]. Furthermore, neither absorption nor total cross section data were included in the analysis, but are nevertheless predicted accurately. Dirac phenomenology also provides good predictions for proton absorption cross sections, but its calculations for both ${ }^{12} \mathrm{C}$ and ${ }^{40} \mathrm{Ca}$ appear to be slightly too large. Below about $150 \mathrm{MeV}$ the Schwandt model also agrees with the data, but its energy dependence appears to be unreasonable and it begins to diverge from the data for higher energies. The earlier version of that model due to Nadasen [78 gives a better description of the energy dependence of the absorption cross section.

In Fig. 2 predictions for neutron total cross sections are compared with the high-quality neutron total cross section data recently obtained at LAMPF [79]. Neutron total cross sections computed from the Schwandt potential are substantially larger than the data. For self-conjugate targets Dirac phenomenology provides good predictions over broad ranges of mass and energy, but, lacking a parametrization of the symmetry potential, the EDAD1 model fails to reproduce $\sigma_{n}$ for $N>Z$ [80. As for the proton absorption cross section, EDAD1 predictions for the ${ }^{12} \mathrm{C}$ neutron total cross sections appear to be slightly too large in the energy range relevant to existing proton knockout data for discrete states. The EEI model also provides good predictions for self-conjugate targets and appears to be more accurate than EDAD1 for light nuclei, such as ${ }^{12} \mathrm{C}$. The result at $200 \mathrm{MeV}$ appears to be slightly too small, as also observed in the proton absorption cross sections. Below about below $300 \mathrm{MeV}$, the EEI calculations for heavier targets with significant neutron excesses also appear to be more accurate than EDAD1, but tend to be too high at higher energies, particularly for ${ }^{208} \mathrm{~Pb}$. For these EEI calculations the symmetry potential is obtained by folding the isovector density with the density-independent isovector interaction from the FL $t$-matrix. Although the isovector interaction has not been calibrated to nucleon-nucleus scattering data with the same care as the isoscalar interaction, its contribution is small enough that residual errors in theoretical models of that term should not be too serious. On the other hand, even though the EEI model provides a good fit to proton elastic scattering by heavy nuclei, there may still be some inaccuracy for large $A$ because the model was fitted to data for $A \leq 40$.

We find that the IA2 interaction provides accurate predictions for elastic and inelastic scattering for $500 \mathrm{MeV}$ protons, where the IA2 interaction is most similar to the empirical effective interaction, but that the real-central repulsion of the IA2 model is too strong 
at lower energies. Nevertheless, Fig. 2 shows that very accurate predictions are obtained for neutron total cross sections [9]. Similarly, calculations using the relativistic impulse approximation, with [32] or without 81] density dependence, also reproduce the the neutron total cross section data for ${ }^{208} \mathrm{~Pb}$. Thus, it will be of interest to compare IA2 predictions with measurements of nuclear transparency at higher energies soon to be made at CEBAF.

In Figs. 1 and 2 we also show calculations based upon the Paris-Hamburg (PH) interaction. Although the $\mathrm{PH}$ optical potential provides good fits to proton elastic scattering data 67,82, we find that its predictions for integrated cross sections are substantially larger than the data. Similarly, PH calculations for proton inelastic scattering to states with surfacepeaked transition densities also tend to produce cross sections that are too large [1, 77, 5]. We have argued [2] that for finite nuclei nonlocal corrections to the LDA suppress the interaction strength in the surface region, producing smaller inelastic cross sections for surface-peaked states and smaller integrated cross sections. This effect is included in the empirical effective interaction and accounts in part for the improvement of the EEI with respect to the PH model for these quantities. Nevertheless, the PH model provides equal or better fits to proton elastic scattering. Thus, there is no guarantee that optical models fitted to elastic scattering data alone will provide the best predictions for absorption or total cross sections or for nuclear transparency. Phenomenological models, such as EEI, which include data that are sensitive to the interior wave function, such as proton inelastic scattering, should provide more accurate interior potentials and better predictions for nuclear transparency. Furthermore, we have also shown that cross sections for exclusive $\left(e, e^{\prime} p\right)$ reactions to discrete final states correlate well with absorption or total cross sections for nucleon-nucleus scattering [9].

\section{RESULTS}

\section{A. Comparison with data for $T_{p} \approx 180 \mathrm{MeV}$}

The first measurements of nuclear transparency for $\left(e, e^{\prime} p\right)$ with $150 \leq T_{p} \leq 210 \mathrm{MeV}$ were made by Garino et al. [10,11] using a $780 \mathrm{MeV}$ electron beam, $\omega=215 \pm 20 \mathrm{MeV}$, and $q \approx 610 \mathrm{MeV} / \mathrm{c}$. Measurements were made for four in-plane opening angles between about $0^{\circ}$ and $23^{\circ}$ with $\phi=180^{\circ}$, which sample a slice through the Fermi cone for this $q$. The ratios

$$
\mathcal{R}(\theta)=\frac{\int d E_{m} d^{5} \sigma\left(e, e^{\prime} p\right) / d E_{m} d \Omega_{e} d \Omega_{p}}{d^{2} \sigma\left(e, e^{\prime}\right) / d \Omega_{e}}
$$

integrated over a wide range of missing energy (up to about $100 \mathrm{MeV}$ ) were measured for ${ }^{12} \mathrm{C},{ }^{27} \mathrm{Al},{ }^{58} \mathrm{Ni}$, and ${ }^{181} \mathrm{Ta}$. Several methods were used to relate the measured ratios $\mathcal{R}(\theta)$ to the nuclear transparency, but the variations were less than $5 \%$, which can be viewed as

an estimate of the systematic error. From our point of view, the experimental realization of Eq. (11) would be

$$
\mathcal{T}_{\perp}=\frac{\int d \theta \sin \theta \mathcal{R}^{D W}(\theta)}{\int d \theta \sin \theta \mathcal{R}^{P W}(\theta)}
$$


where the range of integration is restricted to the large angle side of $\mathbf{q}$. The data for $E_{m} \leq 80$ $\mathrm{MeV}$ are shown in Fig. 3 .

Since this experiment was performed with a relatively narrow acceptance in electron energy, the calculations were performed with fixed $\omega=215 \mathrm{MeV}$ and $q=605 \mathrm{MeV} / \mathrm{c}$. The proton spectrometer, on the other hand, accepted a wide range of proton energies centered about $180 \mathrm{MeV}$. Hence, for each shell the laboratory proton energy for parallel kinematics was computed as $T_{p}=\omega-E_{m}$ where $E_{m}$ is the Hartree-Fock single-particle energy. The invariant mass for each final state was held constant, so that the laboratory proton energies for nonparallel kinematics are slightly smaller. The optical potential for each shell was calculated using the proton energy for that shell.

Unfortunately, the EEI interaction is only available for discrete energies and the parameters do not vary as smoothly as one might like because those interactions were obtained by fitting uncorrelated data sets independently. No attempt has yet been made to impose a smooth energy dependence upon the empirical effective interaction. Nevertheless, the interactions do vary smoothly enough that calculations made using the $180 \mathrm{MeV}$ or the 200 $\mathrm{MeV}$ interaction are quite similar and the small differences between them do not affect the conclusions. Furthermore, a large part of the energy dependence of the effective interaction arises from the exchange contributions, which were evaluated using the kinematics of each orbital. Therefore, we chose to use the fit to data for $180 \mathrm{MeV}$ protons, representing the center of the proton energy distribution. Similar calculations using the $200 \mathrm{MeV}$ EEI interaction, fixed $\omega$ rather than fixed $T_{p}$, and a other few technical differences, were presented in Ref. [9]. Although slightly better agreement with the data was obtained using that slightly less absorptive interaction, the small difference between the two calculations demonstrates that the results are relatively insensitive to uncertainties in the EEI parametrization and ambiguities in the prescription for acceptance averaging.

We have studied the sensitivity of nuclear transparency to the optical model by comparing the EEI model with Dirac phenomenology, version EDAD1. Although the data extend in both energy and mass beyond their ranges of applicability, we also show calculations based upon the Schwandt and the Comfort and Karp (CK) optical potentials for comparison with other authors who have used those models. Ejectile wave functions for the Schwandt and CK potentials include Perey factors, Eq. (22), with $\beta=0.85 \mathrm{fm}$, whereas wave functions based upon Schrödinger-equivalent potentials from Dirac phenomenology include the Darwin factor, Eq. (25). Nonlocality corrections are not needed for the EEI model because both elastic and inelastic scattering are fitted self-consistently in that model. These calculations are compared with the data in Fig. 3. We find that the EEI model provides a good description of nuclear transparency, whereas considerably smaller transparencies are obtained with either the Schwandt or the EDAD1 potentials. These results are consistent with the observation in Sec. III that the EEI model provides more accurate predictions for proton absorption and neutron total cross sections also. It is clear that the EDAD1 model is too absorptive at these energies, so that spectroscopic factors using it will be artificially large to compensate for excessive attenuation.

Similar calculations have been performed by Ireland et al. 83 using the Schwandt potential and they obtained larger transparencies which agree better with the data than do our ostensibly similar calculations with the same potential. However, that analysis suffers from several defects. First, the kinematics were artificially altered so that knockout from 
every orbital was assigned the same ejectile energy, $180 \mathrm{MeV}$, despite the wide range of energies covered by the experiment. Thus, they used the Schwandt potential at $180 \mathrm{MeV}$ even though, for the same electron kinematics, protons ejected from less bound orbitals actually emerged with energies beyond the range of that optical model. Second, their calculations used a scattered electron energy that is $30 \mathrm{MeV}$ higher than the center of the experimental acceptance. Third, the DWIA cross section was computed using a current operator based upon nonrelativistic reduction to second order in $q / m_{N}$, but the distorted momentum distribution was obtained by dividing the cross section by $\sigma_{c c 1}$, the cross section for the $c c 1$ current operator. The inconsistency between the numerator and the denominator in their application of Eq. (2) leads to a spurious enhancement of the transparency above unity for a plane--wave calculation. However, none of these defects appears to be sufficient to explain the discrepancy between the two calculations. Nevertheless, we consider the apparent agreement between the data and the Schwandt calculations of Ref. [83 to be a fortuitous result of an incorrect calculation.

Phenomenological optical models, nonrelativistic or relativistic, which are fitted only to elastic scattering data may fit that data well but still fail to predict nuclear transparency correctly because such analyses are not sensitive to the interior wave function. The EEI model is much more sensitive to the interior wave function because in fitting inelastic scattering data it requires consistency between distorted waves and inelastic transition amplitudes. Therefore, the EEI model provides a more accurate prediction of nuclear transparency and should also provide more accurate spectroscopic factors. Clearly it will be of interest to obtain transparency data for a wider range of energies and such studies are planned for CEBAF. It would also be of interest to obtain comparable data at lower energies where NIKHEF has performed its extensive survey of spectroscopic factors for complex nuclei [9], but it appears that the lower-energy regime will soon be abandoned.

\section{B. Comparison with data for $T_{p} \approx 650 \mathrm{MeV} / \mathrm{c}$}

Nuclear transparency data for $1.0 \leq Q^{2} \leq 6.8(\mathrm{GeV} / \mathrm{c})^{2}$ have recently been obtained at SLAC by the NE18 collaboration [22,23]. In Fig. 团 we compare calculations with the data for $Q^{2}=1.04(\mathrm{GeV} / \mathrm{c})^{2}$ and $\omega=0.625 \mathrm{GeV}$. The angular integrations were symmetric with respect to q. Since the dependence of nuclear transparency on proton energy is quite slow for these energies (see following section), we performed the calculations using a fixed proton energy of $650 \mathrm{MeV}$ for which both the EEI and IA2 interactions are available. The electron kinematics were then computed using $\omega=E_{m}+T_{p}$ for each shell. At this energy we find that the EEI, IA2, and EDAD1 potentials all give practically the same results - the variation due to choice optical potential is much smaller at 650 than at $180 \mathrm{MeV}$. However, although the calculations are fairly close to the data for ${ }^{12} \mathrm{C}$, the data vary much less with $A$ than do the calculations. O'Neill et al. report that the data can be fitted with a power law of the form $A^{-\alpha}$ with $\alpha=0.18$ at $Q^{2}=1.0(\mathrm{GeV} / \mathrm{c})^{2}$ and with slightly larger values of $\alpha$ at higher $Q^{2}$. However, the calculations are much closer to the characteristic $\alpha=1 / 3$ behavior that would be expected for an eikonal model with constant attenuation length.

The experimental values of $\mathcal{T}_{\perp}$ reported by the NE18 collaboration [22,23] include corrections for the portion of the model spectral functions which would fall outside their ac- 
ceptances. These correction factors are 1.11, 1.22, and 1.28 for carbon, iron, and gold, respectively. The calculations of Nikolaev et al. [24 appear to be in good agreement with the $A$-dependence of the data for $Q^{2} \approx 1.0(\mathrm{GeV} / \mathrm{c})^{2}$, but their figure apparently employs a preliminary analysis of the data or neglects these correction factors resulting in smaller experimental values. However, elimination of the correction factors would not be enough to bring the data into agreement with our calculations. As discussed in Sec. \, the most important difference between our calculations and those of Nikolaev et al. can be traced to differences between the definitions of the semi-inclusive cross section from which transparencies are calculated. Nevertheless, it would be of interest to compare proton absorption and neutron total cross sections computed with their Glauber model with nucleon-nucleus data for this energy regime.

\section{Energy Dependence of Nuclear Transparency}

The energy dependence of nuclear transparency is examined in Fig. 5, which compares calculations using the EEI, IA2, and EDAD1 potentials for representative light $\left({ }^{12} \mathrm{C}\right)$, medium $\left({ }^{58} \mathrm{Ni}\right)$, and heavy $\left({ }^{208} \mathrm{~Pb}\right)$ nuclei. The calculations were performed for proton energies marked by symbols, where circles are for ${ }^{12} \mathrm{C}$, squares are for ${ }^{58} \mathrm{Ni}$, and diamonds are for ${ }^{208} \mathrm{~Pb}$. The calculations were made for constant $(\omega, q)$ kinematics using a beam energy of $2.1 \mathrm{GeV}$, although the results are practically independent of the electron energy. The energy transfer for each shell was taken to be $\omega=E_{m}+T_{p}$, where $T_{p}$ is the laboratory kinetic energy for zero recoil and $E_{m}$ is the Hartree-Fock energy for the shell. The angular integrations were symmetric with respect to $\mathbf{q}$.

The proton energies were selected according to the availability of EEI and/or IA2 interactions. The small kinks in the EEI calculations between about 180 and $200 \mathrm{MeV}$ probably indicate the degree of model dependence that arises when fitting independent data sets without imposing a smooth energy dependence. These kinks are clearly correlated with the corresponding neutron total cross section calculations shown in Fig. 2, which suggest that the $200 \mathrm{MeV}$ empirical effective interaction is not quite sufficiently absorptive. On the other hand, the EEI calculations of neutron total cross sections for heavy nuclei tend to be a little too high at $180 \mathrm{MeV}$. Hence, the best estimate of the transparency between 180 and 200 $\mathrm{MeV}$ probably lies between the calculations shown in Fig. 5, with an uncertainty comparable to the difference between them.

For energies below about $200 \mathrm{MeV}$, the EEI model is more transparent than any of the other optical models we have examined and provides the most accurate predictions for the MIT data. Nevertheless, those data still remain about 5-10\% above the EEI calculations. At higher energies the variation among optical potentials tends to decrease, although for medium and heavy nuclei the IA2 calculations are more transparent than either the EEI or EDAD1 models. This behavior is also clearly correlated with the neutron total cross section calculations shown in Fig. 2, where for medium and heavy nuclei the IA2 interaction provides accurate predictions while the EDAD1 and EEI calculations are similar and are both slightly too large.

A series of ${ }^{12} \mathrm{C}\left(e, e^{\prime} p\right)$ experiments performed at MIT-Bates in parallel kinematics near the quasifree ridge between $0.14 \lesssim Q^{2} \lesssim 0.83(\mathrm{GeV} / \mathrm{c})^{2}$ shows that there is a significant, 
nearly constant, continuum yield extending to very large missing energies even when the missing momentum is relatively small. The continuum yield at large $E_{m}$ is predominantly transverse and cannot be reproduced by calculations of multiple scattering in the final state. A review of these data may be found in Ref. [9]. One possible interpretation of these results is that multinucleon absorption of the virtual photon enhances the yield at large missing energies even when the missing momentum is relatively small. Since the present model does not include multinucleon absorption mechanisms, perhaps it is not surprising that DWA calculations underestimate the semi-inclusive cross section for ${ }^{12} \mathrm{C}\left(e, e^{\prime} p\right)$. For $\omega=215 \mathrm{MeV}$ the ratio between experiment and calculation is approximately constant, but for $\omega \approx 650 \mathrm{MeV}$, the discrepancy increases with $A$. Although no calculations of multinucleon absorption are available for these kinematics, it seems reasonable to assume that such effects can become more important as either $A$ or $\omega$ increases such that more energy is available to be shared among more nucleons at greater average density. Furthermore, Lourie et al. [84] have suggested that kinematic focussing of the multinucleon phase space would exacerbate the artificial enhancement of semi-inclusive transparency measurements as $Q^{2}$ increases.

\section{DISCUSSION}

Most previous calculations of nuclear transparency have been based upon eikonal models. For example, Pandharipande and Pieper [12] studied nuclear transparency using a correlated Glauber model in which

$$
\begin{aligned}
\mathcal{T} & =\frac{1}{Z} \int d^{3} r^{\prime} \rho_{p}\left(\mathbf{r}^{\prime}\right) P_{T}\left(\mathbf{r}^{\prime}\right) \\
P_{T}\left(\mathbf{r}^{\prime}\right) & =\exp \left\{-\int_{z^{\prime}}^{\infty} d z^{\prime \prime}\left[g_{p n}\left(\mathbf{r}^{\prime}, \mathbf{r}^{\prime \prime}\right) \tilde{\sigma}_{p n}\left(q, \rho\left(\mathbf{r}^{\prime \prime}\right)\right) \rho_{n}\left(\mathbf{r}^{\prime \prime}\right)+g_{p p}\left(\mathbf{r}^{\prime}, \mathbf{r}^{\prime \prime}\right) \tilde{\sigma}_{p p}\left(q, \rho\left(\mathbf{r}^{\prime \prime}\right)\right) \rho_{p}\left(\mathbf{r}^{\prime \prime}\right)\right]\right\}
\end{aligned}
$$

where $P_{T}\left(\mathbf{r}^{\prime}\right)$ represents the probability that a proton struck at position $\mathbf{r}^{\prime}$ will emerge without rescattering, $\tilde{\sigma}_{p N}\left(q, \rho\left(\mathbf{r}^{\prime \prime}\right)\right)$ represents the effective $p N$ cross section evaluated in local density approximation, and

$$
g_{p N}\left(\mathbf{r}^{\prime}, \mathbf{r}^{\prime \prime}\right) \approx g_{p N}\left(\rho_{0},\left|\mathbf{r}^{\prime}-\mathbf{r}^{\prime \prime}\right|\right)
$$

represents the pair distribution function evaluated at central density. Although this calculation agrees well with the MIT data, it should be noted that Eq. (30) applies to $\mathcal{T}_{\|}$ whereas the experiment measured $\mathcal{T}_{\perp}$. It is also important to recognize that despite the differences between the formulations of these models, the EEI model includes essentially the same physics. Recall that the EEI parametrization was originally based upon BruecknerHartree-Fock calculations of the effective interaction in nuclear matter which include both short-range correlations and Pauli blocking, but that the parameters were adjusted to improve the fit to experimental data. The correlated Glauber model includes Pauli blocking in its effective cross section, whereas the EEI model includes it as a density-dependent damping of its imaginary central interaction. Similarly, the anticorrelation between identical nucleons is represented by the pair distribution function in the correlated Glauber model or by 
the density-dependent short-ranged repulsion in the real central EEI interaction. Pandharipande and Pieper find that Pauli blocking, effective mass, and correlation effects all play important roles in their transparency calculations.

However the validity of the Glauber model is questionable at low energies where rectilinear propagation is a poor approximation, particularly for energies as low as $180 \mathrm{MeV}$ used in the MIT experiment and the corresponding calculations of Ref. [12]. The Glauber approximation also uses a zero-range approximation to the nucleon-nucleon interaction, which does not apply at intermediate energies even if Pauli blocking and correlation corrections are made. The local density approximation provides a more realistic description of the radial dependence of the optical potential, but includes the Glauber approximation as a limiting case. For proton elastic scattering the Glauber model is generally considered adequate for $T_{p} \gtrsim 800 \mathrm{MeV}$ 14, 15. Although integrated quantities, such as nuclear transparency, are probably less sensitive to the details of final state interactions, the lowest energy at which the Glauber approximation to nuclear transparency can be employed has not been established. On the other hand, the Glauber approximation is more efficient than the distorted wave approximation and should become sufficiently accurate for $T_{p} \gtrsim 800 \mathrm{MeV}$. Furthermore, we find that the variation among optical potentials also decreases at higher energies where the Glauber approximation becomes applicable. Therefore, Glauber calculations are more appropriate for $Q^{2} \gtrsim 2.0(\mathrm{GeV} / \mathrm{c})^{2}$.

Neither the optical nor the Glauber model consider in detail the distribution of absorbed flux among final states of the residual system. To the extent that nucleon knockout dominates the absorption cross section at intermediate energies, most of the flux leaving the elastic channel leads to multinucleon final states in the continuum which should be excluded from the semi-inclusive cross section used to measure nuclear transparency. Of course, it is much easier to exclude the multinucleon continuum theoretically than experimentally. Inelastic processes within final-state interactions that result in single-hole states of the residual nucleus would require a coupled-channels model for detailed analysis and should be included in the semi-inclusive cross section, but represent a rather small fraction of the absorption cross section. Therefore, we expect that most inelastic processes reduce transparency as stipulated by the optical model and the distorted wave approximation.

Nuclear transparency for $\left(e, e^{\prime} p\right)$ at large $Q^{2}$ is also usually interpreted using a Glauber model. As developed by Nikolaev et al. [30,85], the Glauber transparency functions for parallel and quasiperpendicular kinematics take the form

$$
\begin{aligned}
& \mathcal{T}_{\|}^{G}=\frac{1}{A \sigma_{\text {tot }}(p N)} \int d^{2} b\left\{1-\exp \left[-\sigma_{\text {tot }}(p N) t(b)\right]\right\} \\
& \mathcal{T}_{\perp}^{G}=\frac{1}{A \sigma_{\text {in }}(p N)} \int d^{2} b\left\{1-\exp \left[-\sigma_{\text {in }}(p N) t(b)\right]\right\}
\end{aligned}
$$

where

$$
t(b)=\int_{-\infty}^{\infty} d z n_{A}(b, z)
$$

is the optical thickness at impact parameter $b$ through nuclear density $n_{A}(b, z)$. These results for nuclear transparency in the Glauber model are distinguished from our definitions by the superscript $G$. The transparency for parallel kinematics, $\mathcal{T}_{\|}^{G}$, is governed by the 
total proton-nucleon cross section, $\sigma_{t o t}(p N)$, which represents the loss of flux in the forward direction (parallel to the momentum transfer). The transparency for quasiperpendicular kinematics is larger because integration over the full angular range recaptures the part of the flux that corresponds to elastic proton-nucleon final-state interactions and hence $\mathcal{T}_{\perp}^{G}$ is governed by the inelastic proton-nucleon cross section, $\sigma_{i n}(p N)$.

Nikolaev et al. 224 demonstrated that the difference between $\mathcal{T}_{\|}^{G}$ and $\mathcal{T}_{\perp}^{G}$ is quite large at low $Q^{2}$ where the inelasticity of the nucleon-nucleon interaction is relatively small. Furthermore, averaging over the acceptance for experiment NE18, they obtained an accurate description of the data that is intermediate between $\mathcal{T}_{\perp}^{G}$ and $\mathcal{T}_{\|}^{G}$. However, for $Q^{2}<1$ $(\mathrm{GeV} / \mathrm{c})^{2}$ the difference between $\mathcal{T}_{\|}$and $\mathcal{T}_{\perp}$ is much smaller in the distorted wave approximation than in the Glauber model (see the end of Sec. IIA). Since the absorptive content of the optical potential for energies below the pion production threshold is determined by quasifree elastic nucleon-nucleon scattering, one expects $\mathcal{T}_{\|}^{G} \approx \mathcal{T}_{\|} \approx \mathcal{T}_{\perp}$ for energies large enough to neglect Pauli blocking. On the other hand, the most important reason why the Glauber model calculations of Nikolaev et al. [24] produce larger transparencies than our optical models for $Q^{2} \gtrsim 1(\mathrm{GeV} / \mathrm{c})^{2}$ can be traced to differences between the definitions of the semi-inclusive cross sections used to derive $\mathcal{T}_{\perp}^{G}$ and $\mathcal{T}_{\perp}$ that arise from differences between a high-energy versus a low-energy viewpoint.

The derivation of Eq. (31b) requires a closure sum over all final states of the residual nuclear system that contain $A-1$ nucleons (e.g. Ref. [85]). Thus, the semi-inclusive cross section used for $\mathcal{T}_{\perp}^{G}$ includes processes in which one or more secondary nucleons is ejected from the residual nucleus by final-state interactions with the primary ejectile even if the resultant missing energy is outside the experimental acceptance. From a high-energy viewpoint, such processes are described as incoherent elastic rescatterings of the ejectile and are driven by the elastic proton-nucleon cross section, $\sigma_{e l}(p N)$. In fact, the only final states excluded from the semi-inclusive cross section for $\mathcal{T}_{\perp}^{G}$ are those which contain additional particles, typically one or more pions for modest $\omega$. Therefore, in the absence of Fermi motion, $\mathcal{T}_{\perp}^{G}$ for ejectile energies below the pion production threshold would be unity because the proton-nucleon interaction is then elastic. According to Table 1 of Ref. [85], $\mathcal{T}_{\perp}^{G} \approx 0.97$ for ${ }^{12} \mathrm{C}$ or $\mathcal{T}_{\perp}^{G} \approx 0.84$ for ${ }^{208} \mathrm{~Pb}$ remains large even for $Q^{2} \sim 1(\mathrm{GeV} / \mathrm{c})^{2}$. For larger ejectile energies $\mathcal{T}_{\perp}^{G}$ decreases as the proton-nucleon interaction becomes increasing inelastic and approaches $\mathcal{T}_{\|}^{G}$ for large $Q^{2}$.

By contrast, the semi-inclusive cross section used to obtain $\mathcal{T}_{\perp}$ within the optical model excludes multinucleon emission arising from final-state interactions. From a low-energy viewpoint, nuclear inelastic scattering which changes the internal state of the residual nucleus is described as a loss of flux from the elastic channel. Thus, inelastic scattering involving a small energy transfer between the ejectile and the residual contributes to the absorptive (imaginary) potential even if the final state remains a low-lying excitation of the $(A-1)$ system. Since some of these low-lying final states fall within the missing-energy acceptance and are thereby included in the experimental semi-inclusive cross section, optical model calculations using potentials fitted to elastic nucleon-nucleus scattering probably slightly overestimate the loss of flux. More accurate models which account for this effect would require coupled-channels calculations based upon optical potentials constructed for the model space included within the semi-inclusive cross section, but, fortunately, since the imaginary 
part of the optical potential for intermediate energy nucleons with $100 \lesssim T_{p} \lesssim 800 \mathrm{MeV}$ is dominated by quasifree knockout processes, this error is small and decreases as the ejectile energy increases. Nevertheless, it is clear that the more restrictive summation over final states in the optical model definition of nuclear transparency must result in $\mathcal{T}_{\perp}<\mathcal{T}_{\perp}^{G}$. Furthermore, the optical model definition of nuclear transparency is more relevant to exclusive $\left(e, e^{\prime} p\right)$ measurements of the missing-momentum distributions for discrete final states and to semi-inclusive experiments of the type performed by MIT and illustrated in Fig. 3, for which the Glauber model would give a result, $\mathcal{T}_{\perp}^{G} \approx 1$, that is much larger than the data because the ejectile energy is below the nucleon-nucleon inelasticity threshold. Although several other assumptions employed in its derivation also fail and preclude its application below about $1(\mathrm{GeV} / \mathrm{c})^{2}$, the most important deficiency of this version the Glauber model for low $Q^{2}$ is that its summation over final states is much more inclusive than the experimental definition of the semi-inclusive cross section.

The Glauber model for $\mathcal{T}_{\perp}^{G}$ emphasizes the inelasticity of the nucleon-nucleon interaction, whereas the optical model for $\mathcal{T}_{\perp}$ emphasizes the inelasticity of the nucleon-nucleus interaction. Those final-state interactions which eject one or more low-energy nucleons but leave the missing energy within the integration range of the experiment reduce $\mathcal{T}_{\perp}$ but do not reduce $\mathcal{T}_{\perp}^{\text {exp }}$. On the other hand, elastic proton-nucleon interactions which increase the missing energy too much reduce $\mathcal{T}_{\perp}^{\text {exp }}$ but do not reduce $\mathcal{T}_{\perp}^{G}$. Therefore, the experimental definition of the semi-inclusive cross section for $\left(e, e^{\prime} p\right)$ reactions lies between those used for $\mathcal{T}_{\perp}^{G}$ and $\mathcal{T}_{\perp}$. For relatively low $Q^{2}$ the optical model is most appropriate, but will generally underestimate the transparency whereas the Glauber model for $\mathcal{T}_{\perp}$ will substantially overestimate the transparency. For large $Q^{2}$ where the reactive content of the optical potential is dominated by nucleon-nucleon inelasticity we would expect $\mathcal{T}_{\perp}$ to approach $\mathcal{T}_{\perp}^{G}$ from below, but the Glauber model is clearly much more efficient computationally than the optical model under these conditions. In between we would expect these two models to bracket the data. However, neither approach includes multinucleon absorption of the virtual photon upon a correlated cluster, which also might increase the experimental cross section with respect to direct knockout models.

Experimentally it would be interest to observe multinucleon knockout by electron scattering and to study the kinematic dependencies of various multinucleon channels in detail. Although it is not possible to separate the various processes which lead to the same final state in a model independent fashion, the kinematic differences among them can be usefully analyzed in the context of a model. For example, single-nucleon knockout from a deeply bound orbital leaves the residual nucleus in a highly excited state which may decay by particle emission and hence produce multinucleon emission without need of final-state interactions. Multinucleon processes of this type are probably close to isotropic in the rest frame of the residual nucleus and from an optical-model viewpoint should be included in the semi-inclusive cross section because final-state interactions are not required. On the other hand, one might expect secondary nucleons knocked out by interactions with the ejectile to appear preferentially in the forward hemisphere; such events should be included in the cross section for $\mathcal{T}_{\perp}^{G}$ but excluded for $\mathcal{T}_{\perp}$. Analyses of this type require measurement of the angular correlation between the high-energy primary proton and one or more low-energy secondary nucleons. Similarly, although it is not possible to unambiguously identify events arising from multinucleon absorption of the virtual photon upon a correlated cluster, an 
enhancement of the probability for observing two nucleons corresponding to missing momenta $\pm p_{m}$ with $p_{m} \gtrsim k_{F}$ can be interpreted within a model as a signature of short-range correlations. Although one would expect the longitudinal/transverse character of multinucleon emission arising from the decay of a deep-hole state or from final-state interactions to remain consistent with the direct knockout model, multinucleon absorption of the virtual photon could substantially alter the structure of the response functions. In fact, there is some evidence at low $Q^{2}$ that nucleon knockout at large missing energy is enhanced by a process, perhaps multinucleon absorption, that is largely transverse. However, clarification of these issues will require considerably more work, both experimental and theoretical.

It may be possible to refine optical model calculations of nuclear transparency by applying

the statistical multistep direct reaction theory of Feshbach, Kerman, and Koonin [86] to evaluate the energy and angular distributions of protons which suffer final state interactions and thereby to estimate the fraction of the flux described by the optical model as absorption that actually remains within the experimental acceptances. However, it would then also be necessary to test those calculations against inclusive data for $\left(p, p^{\prime}\right)$. Such data is available at low energies, e.g. Refs. 87,88, but is not available for $T_{p}>200 \mathrm{MeV}$. If data were available, it should also be possible to estimate the necessary corrections using a convolution procedure. However, these possibilities lie well beyond the scope of the present work.

\section{CONCLUSIONS}

We have used the distorted wave approximation to evaluate nuclear transparency to intermediate-energy protons in semi-inclusive $\left(e, e^{\prime} p\right)$ reactions. We compared calculations using density-dependent effective interactions from the EEI model, which is fitted to proton elastic and inelastic scattering data, and the IA2 model, which is derived from a relativistic boson exchange model, with global optical potentials from Dirac phenomenology. For low energies we also considered several traditional nonrelativistic optical models. We demonstrated that nuclear transparency in $\left(e, e^{\prime} p\right)$ reactions is well correlated with the proton absorption and neutron total cross sections calculated using these models. The IA2 model was found to give the most accurate predictions for neutron total cross sections at $T_{p} \gtrsim 200$ $\mathrm{MeV}$. The EEI model also provides accurate predictions and extends to lower energies, but slightly overestimates the neutron total cross sections for heavy nuclei at $T_{p} \gtrsim 300 \mathrm{MeV}$.

For ejectile energies near $200 \mathrm{MeV}$ we find that there is considerable sensitivity to the choice of optical model. Global optical potentials from Dirac phenomenology yield nuclear transparencies that are much smaller than the data, and hence are likely to overestimate spectroscopic factors for discrete states. Larger transparencies are obtained from the IA2 model, but those calculations remain significantly lower than the data. Calculations using the EEI model predict larger transparencies than any other model considered, but still remain 5-10\% below the data. The EEI model predicts greater transparency than other models for $T_{p} \lesssim 200 \mathrm{MeV}$, but low-energy semi-inclusive data are presently lacking. At larger ejectile energies the sensitivity to the choice of optical model is reduced, with all models considered producing similar results for $T_{p} \gtrsim 500 \mathrm{MeV}$. Nevertheless, the calculated nuclear transparencies remain substantially below the NE18 data for $Q^{2} \approx 1(\mathrm{GeV} / \mathrm{c})^{2}$, with the discrepancy increasing with $A$. Multinucleon contributions to the continuum may 
enhance the semi-inclusive cross section for knockout and thereby artficially enhance the measured nuclear transparency. These contributions are likely to increase with both mass and energy, but quantitative estimates are not available.

The present model is well suited to the investigation of intermediate-energy proton knockout to discrete states of the residual nucleus. The dependence of attenuation factors for valence orbitals upon ejectile energy can then be used to investigate nuclear transparency, where differing radial localizations can help discriminate between interior and surface properties of the optical potential. The asymmetry between parallel and antiparallel kinematics for individual orbitals can be investigated also. Recoil polarization may also provide additional insight into the final-state interactions, and in particular may help to discriminate between single-nucleon and multinucleon contributions to the continuum.

We have also examined in some detail the difference between Glauber and optical model calculations of nuclear transparency for quasiperpendicular kinematics. By using a much more inclusive summation over final states, the Glauber model emphasizes the inelasticity of the nucleon-nucleon interaction, whereas with a more restrictive definition of the semiinclusive cross section the optical model emphasizes the role of nucleon-nucleus inelasticity. Therefore, the Glauber model produces larger transparency factors than the optical model, with the difference between the two approaches becoming quite large for $Q^{2}<1(\mathrm{GeV} / \mathrm{c})^{2}$. However, the experimental definition of the semi-inclusive cross section usually lies between these extremes. Although the optical model is expected to underestimate the experimental semi-inclusive cross section, it is much more appropriate for low $Q^{2}$ than the Glauber model. For large $Q^{2}$ where nucleon-nucleon inelasticity accounts for a much larger fraction of the nucleon-nucleus inelasticity, the two models should produce similar results, but the Glauber approximation is computationally much more efficient.

\section{ACKNOWLEDGMENTS}

The support of the U.S. National Science Foundation under grant PHY-9220690 is gratefully acknowledged. 


\section{REFERENCES}

[1] J. J. Kelly et al., Phys. Rev. C 39, 1222 (1989).

[2] J. J. Kelly, Phys. Rev. C 39, 2120 (1989).

[3] J. J. Kelly et al., Phys. Rev. C 41, 2504 (1990).

[4] Q. Chen, J. J. Kelly, P. P. Singh, M. C. Radhakrishna, W. P. Jones, and H. Nann, Phys. Rev. C 41, 2514 (1990).

[5] J. J. Kelly et al., Phys. Rev. C 43, 1272 (1991).

[6] B. S. Flanders et al., Phys. Rev. C 43, 2103 (1991).

[7] A. E. L. Dieperink and P. K. A. de Witt Huberts, Ann. Rev. Nucl. Part. Sci. 40, 239 (1990).

[8] S. Boffi, C. Giusti, and F. D. Pacati, Phys. Rep. 226, 1 (1993).

[9] J. J. Kelly, Adv. Nucl. Phys. 23, 75 (1996).

[10] G. Garino et al., Phys. Rev. C 45, 780 (1992).

[11] D. F. Geesaman et al., Phys. Rev. Lett. 63, 734 (1989).

[12] V. R. Pandharipande and S. C. Pieper, Phys. Rev. C 45, 791 (1992).

[13] R. J. Glauber, in Lectures in Theoretical Physics, edited by W. E. Brittin and L. G. Dunham (Interscience, New York, 1959), Vol. 1, pp. 315-414.

[14] W. H. Bassichis, H. Feshbach, and J. F. Reading, Ann. Phys. (N.Y.) 68, 462 (1971).

[15] L. L. Frankfurt, M. I. Strikman, and M. B. Zhalov, Phys. Rev. C 50, 2189 (1994).

[16] S. J. Brodsky, in Proceedings of the Thirteenth International Symposium on Multiparticle Dynamics, edited by W. Kittel, W. Metzger, and A. Stergiou (World Scientific, Singapore, 1982), p. 963.

[17] A. H. Mueller, in Proceedings of the XVII Recontre de Moriond, 1982, edited by J. Tran Thanh Van (Editions Frontieres, Gif-sur-Yvette, France, 1982), p. 13.

[18] G. R. Farrar, H. Liu, L. L. Frankfurt, and M. I. Strikman, Phys. Rev. Lett. 61, 686 (1988).

[19] L. L. Frankfurt, M. I. Strikman, and M. B. Zhalov, Nucl. Phys. A515, 599 (1990).

[20] B. K. Jennings and G. A. Miller, Phys. Rev. Lett. 69, 3619 (1992).

[21] O. Benhar, A. Fanrocini, S. Fantoni, V. R. Pandharipande, and I. Sick, Phys. Rev. Lett. 69, 881 (1992).

[22] N. C. R. Makins et al., Phys. Rev. Lett. 72, 1986 (1994).

[23] T. G. O'Neill et al., Phys. Lett. B351, 87 (1995).

[24] N. N. Nikolaev, A. Szczurek, J. Speth, J. Wambach, B. G. Zakharov, and V. R. Zoller, Phys. Rev. C 50, R1296 (1994).

[25] N. N. Nikolaev, A. Szczurek, J. Speth, J. Wambach, B. G. Zakharov, and V. R. Zoller, Phys. Lett. B317, 281 (1993).

[26] A. Kohama, K. Yazaki, and R. Seki, Nucl. Phys. A551, 687 (1993).

[27] N. N. Nikolaev, A. Szczurek, J. Speth, J. Wambach, B. G. Zakharov, and V. R. Zoller, Phys. Lett. B317, 287 (1993).

[28] N. N. Nikolaev, A. Szczurek, J. Speth, J. Wambach, B. G. Zakharov, and V. R. Zoller, Nucl. Phys. A567, 781 (1994).

[29] B. K. Jennings and B. Z. Kopeliovich, Phys. Rev. Lett. 70, 3384 (1993).

$[30]$ N. N. Nikolaev, J. Speth, and B. G. Zakharov, preprint (unpublished).

[31] R. J. Furnstahl and S. J. Wallace, Phys. Rev. C 47, 2812 (1993). 
[32] J. J. Kelly and S. J. Wallace, Phys. Rev. C 49, 1315 (1994).

[33] E. D. Cooper, S. Hama, B. C. Clark, and R. L. Mercer, Phys. Rev. C 47, 297 (1993).

[34] J. Friedrich and P.-G. Reinhard, Phys. Rev. C 33, 335 (1986).

[35] P.-G. Reinhard, in Computational Nuclear Physics 1: Nuclear Structure, edited by K. Langanke, J. A. Maruhn, and S. E. Koonin (Springer-Verlag, Berlin, 1991), pp. 28-50.

[36] T. de Forest, Annals of Physics 45, 365 (1967).

[37] S. Boffi, C. Giusti, F. D. Pacati, and S. Frullani, Nucl. Phys. A319, 461 (1979).

[38] L. L. Schiff, Phys. Rev. 103, 443 (1956).

[39] Y. Jin, H. P. Blok, and L. Lapikás, Phys. Rev. C 48, R964 (1993).

[40] T. de Forest, Nucl. Phys. A392, 232 (1983).

[41] M. F. Gari and W. Krümpelmann, Phys. Lett. B274, 159 (1992).

[42] M. F. Gari and W. Krümpelmann, Phys. Lett. B282, 483 (1992).

[43] L. Ray, G. W. Hoffmann, and W. R. Coker, Phys. Rep. 212, 223 (1992).

[44] P. Schwandt, H. O. Meyer, W. W. Jacobs, A. D. Bacher, S. E. Vigdor, M. D. Kaitchuck, and T. R. Donoghue, Phys. Rev. C 26, 55 (1982).

[45] I. Abdul-Jalil and D. F. Jackson, J. Phys. G 5, 1699 (1979).

[46] D. F. Jackson and I. Abdul-Jalil, J. Phys. G 6, 481 (1980).

[47] J. R. Comfort and B. C. Karp, Phys. Rev. C 21, 2162 (1980).

[48] F. Perey and B. Buck, Nucl. Phys. 32, 353 (1962).

[49] F. G. Perey, in Direct Interactions and Nuclear Reaction Mechanisms, edited by E. Clementel and C. Villi (Gordon Breach, N.Y., 1963), pp. 125-138.

[50] Y. Jin and D. S. Onley, Phys. Rev. C 50, 377 (1994).

[51] S. Hama, B. C. Clark, E. D. Cooper, H. S. Sherif, and R. L. Mercer, Phys. Rev. C 41, 2737 (1993).

[52] J. Hüfner and C. Mahaux, Ann. Phys. (N.Y.) 73, 525 (1972).

[53] J. P. Jeukenne, A. Lejeune, and C. Mahaux, Phys. Rev. C 10, 1391 (1974).

[54] J. P. Jeukenne, A. Lejeune, and C. Mahaux, Phys. Rep. 25, 83 (1976).

[55] J. P. Jeukenne, A. Lejeune, and C. Mahaux, Phys. Rev. C 15, 10 (1977).

[56] J. P. Jeukenne, A. Lejeune, and C. Mahaux, Phys. Rev. C 16, 80 (1977).

[57] R. V. Reid, Ann. Phys. (N.Y.) 50, 411 (1968).

[58] F. A. Brieva and J. R. Rook, Nucl. Phys. A291, 299 (1977).

[59] F. A. Brieva and J. R. Rook, Nucl. Phys. A291, 317 (1977).

[60] F. A. Brieva and J. R. Rook, Nucl. Phys. A297, 206 (1978).

[61] F. A. Brieva and J. R. Rook, Nucl. Phys. A307, 493 (1978).

[62] H. V. von Geramb, F. A. Brieva, and J. R. Rook, in Microscopic Optical Potentials, edited by H. von Geramb (Springer-Verlag, Berlin, 1979), p. 104.

[63] T. Hamada and I. D. Johnston, Nucl. Phys. 34, 382 (1962).

[64] P. J. Siemens, Nucl. Phys. A141, 225 (1970).

[65] N. Yamaguchi, S. Nagata, and J. Michiyama, Prog. Theor. Phys. 70, 459 (1983).

[66] N. Yamaguchi, S. Nagata, and J. Michiyama, Prog. Theor. Phys. 76, 1289 (1986).

[67] L. Rikus, K. Nakano, and H. V. von Geramb, Nucl. Phys. A414, 413 (1984).

[68] H. V. von Geramb, in The Interaction Between Medium Energy Nucleons in Nuclei 1982, Vol. 97 of AIP Conference Proceeding, edited by H. Meyer (AIP, New York, 1983), p. 44 .

[69] M. Lacombe, B. Loiseau, J. M. Richard, R. Vinh Mau, J. Côté, P. Pirès, and R. de Tour- 
reil, Phys. Rev. C 21, 861 (1980).

[70] K. Nakayama and W. G. Love, Phys. Rev. C 38, 51 (1988).

[71] R. Machleidt, K. Holinde, and C. Elster, Phys. Rep. 149, 1 (1987).

[72] L. Ray, Phys. Rev. C 41, 2816 (1990).

[73] E. Clementel and C. Villi, Il Nuovo Cimento 2, 176 (1955).

[74] M. A. Franey and W. G. Love, Phys. Rev. C 31, 488 (1985).

[75] H. de Vries, C. W. de Jager, and C. de Vries, At. Data and Nucl. Data Tables 36, 495 (1987).

[76] W. Bauhoff, At. Data Nucl. Data Tables 35, 429 (1986).

[77] H. Seifert et al., Phys. Rev. C 47, 1615 (1993).

[78] A. Nadasen, P. Schwandt, P. P. Singh, W. W. Jacobs, A. D. Bacher, P. T. Debevec, M. D. Kaitchuck, and J. T. Meek, Phys. Rev. C 23, 1023 (1981).

[79] R. W. Finlay, W. P. Abfalterer, G. Fink, E. Montei, T. Adami, P. W. Lisowski, G. L. Morgan, and R. C. Haight, Phys. Rev. C 47, 237 (1993).

[80] R. W. Finlay, G. Fink, W. P. Abfalterer, P. W. Lisowski, G. L. Morgan, and R. C. Haight, in Proceedings of the International Conference on Nuclear Data for Science and Technology, edited by S. M. Qaim (Springer-Verlag, Berlin, 1992), p. 720.

[81] Y. Jin and R. W. Finlay, Phys. Rev. C 47, 1697 (1993).

[82] L. Rikus and H. V. von Geramb, Nucl. Phys. A426, 496 (1984).

[83] D. G. Ireland and G. van der Steenhoven, Phys. Rev. C 49, 2182 (1994).

[84] R. W. Lourie, W. Bertozzi, J. Morrison, and L. B. Weinstein, Phys. Rev. C 47, R444 (1993).

[85] N. N. Nikolaev, A. Szczurek, J. Speth, J. Wambach, B. G. Zakharov, and V. R. Zoller, Nucl. Phys. A582, 665 (1995).

[86] H. Feshbach, A. Kerman, and S. Koonin, Ann. Phys. (N.Y.) 125, 429 (1980).

[87] A. A. Cowley et al., Phys. Rev. C 43, 678 (1991).

[88] S. V. Förtsch, A. A. Cowley, J. J. Lawrie, D. M. Whittal, J. V. Pilcher, and F. D. Smit, Phys. Rev. C 43, 691 (1991). 


\section{FIGURES}

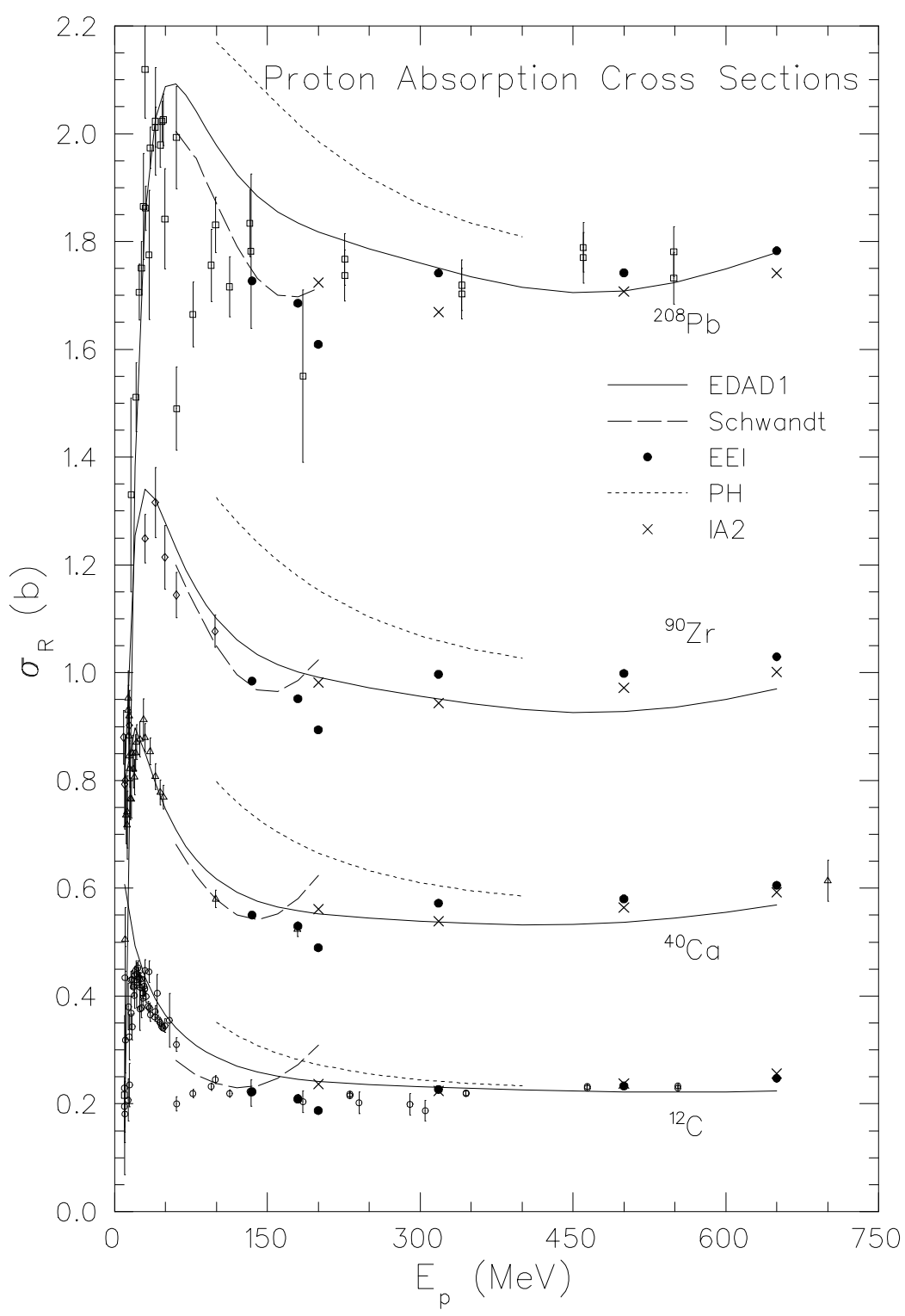

FIG. 1. Proton absorption cross sections for several optical models are compared with data. Note that for ${ }^{12} \mathrm{C}$ and ${ }^{40} \mathrm{Ca}$ the IA2 and EEI calculations sometimes almost coincide and the EEI calculations sometimes obscure data points. 


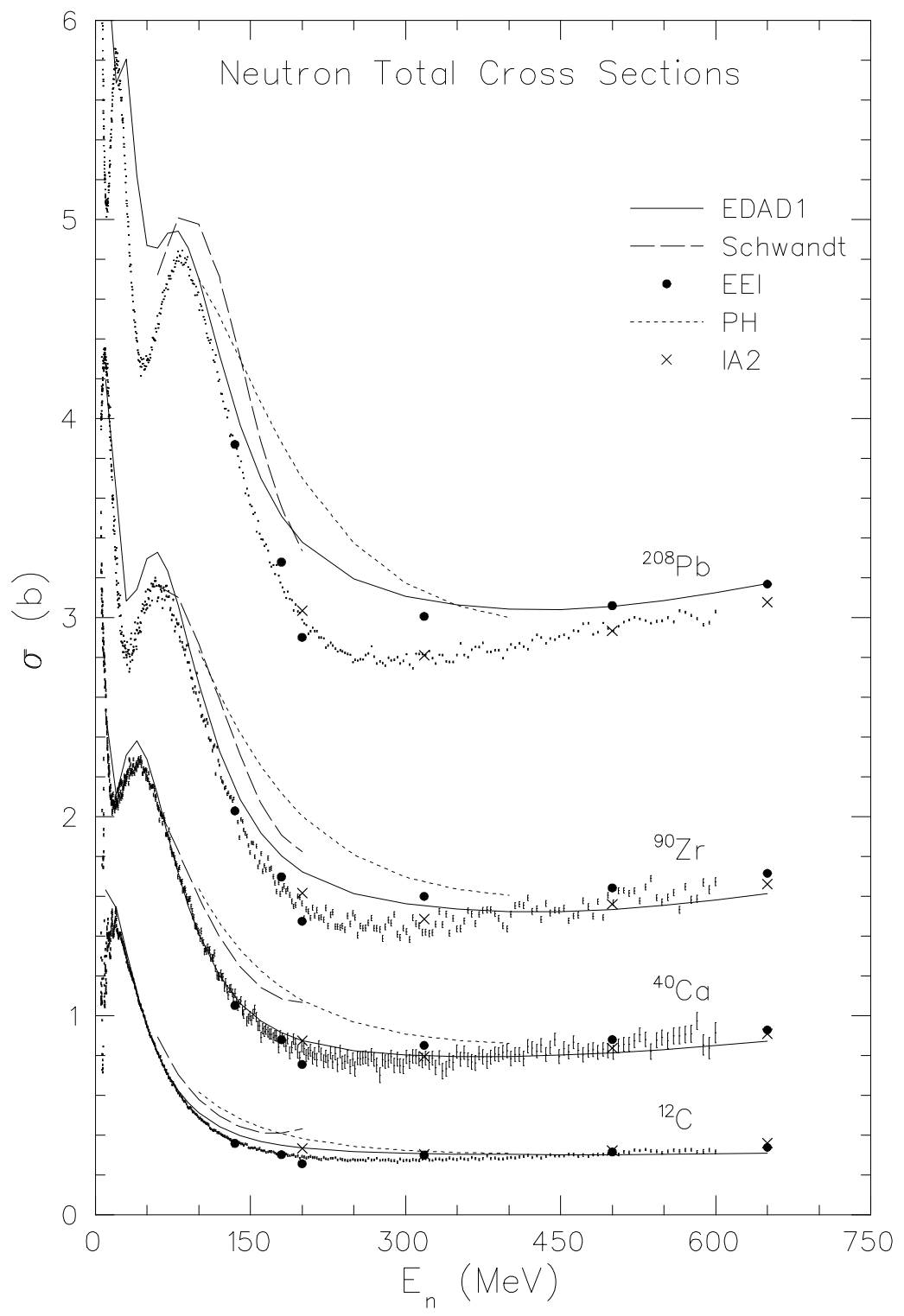

FIG. 2. Neutron total cross sections for several optical models are compared with data. Note that for ${ }^{12} \mathrm{C}$ and ${ }^{40} \mathrm{Ca}$ the IA2 and EEI calculations sometimes almost coincide. 


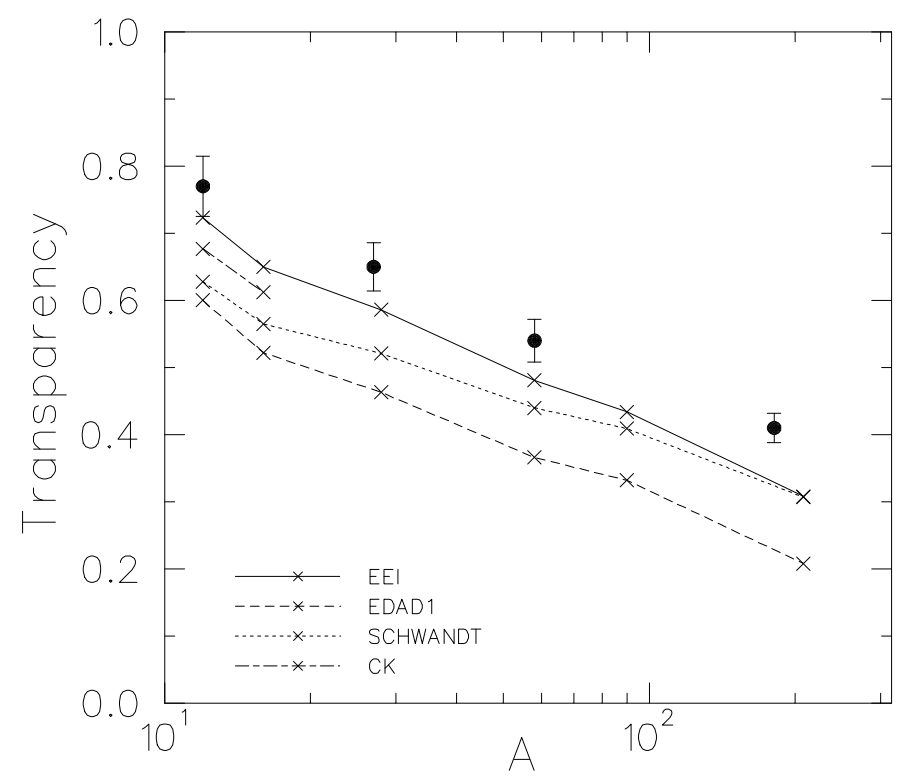

FIG. 3. Nuclear transparency data for $T_{p} \approx 180 \mathrm{MeV}$ are compared with calculations of $\mathcal{T}_{\perp}$ using several optical models. The data (solid points) were obtained using a $780 \mathrm{MeV}$ electron beam, $\omega=215 \pm 20 \mathrm{MeV}$, and $q \approx 605 \mathrm{MeV} / \mathrm{c}$. Calculations were performed for selected closed sub-shell nuclei with mass numbers indicated by crosses. The solid line employs the EEI, dashes the EDAD1, dots the Schwandt, and dash-dots the Comfort and Karp (CK) potential.

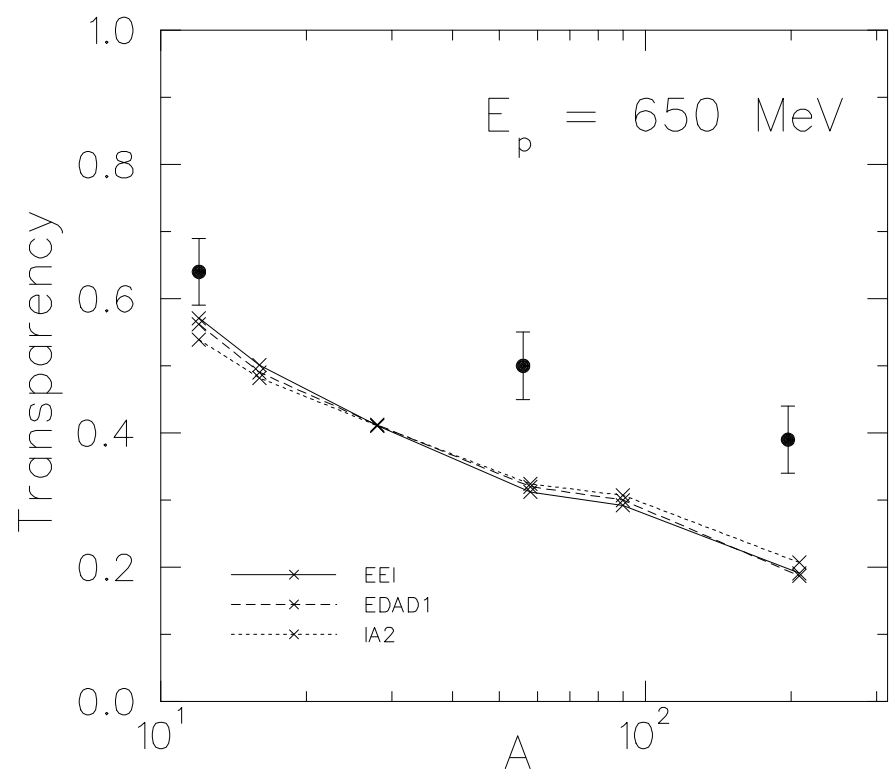

FIG. 4. Nuclear transparency data for $Q^{2}=1.04(\mathrm{GeV} / \mathrm{c})^{2}$ are compared with calculations of $\mathcal{T}_{\perp}$ using several optical models at $T_{p}=650 \mathrm{MeV}$. Calculations were performed for selected closed sub-shell nuclei with mass numbers indicated by crosses. The solid line employs the EEI, dashes the EDAD1, dots the IA2 optical models. 


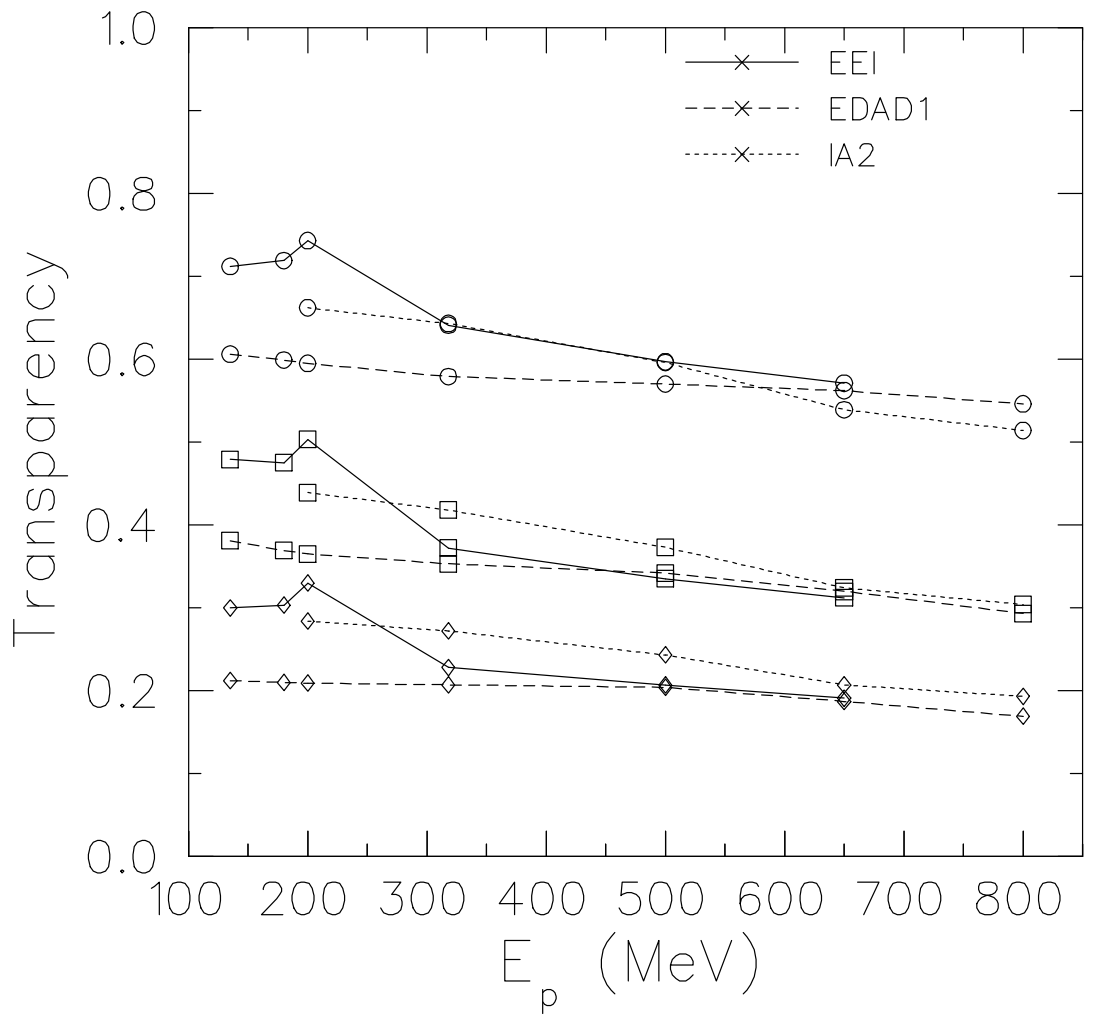

FIG. 5. The energy dependence of nuclear transparency is shown for ${ }^{12} \mathrm{C}$ (circles), ${ }^{58} \mathrm{Ni}$ (squares), and ${ }^{208} \mathrm{~Pb}$ (diamonds) using quasiperpendicular kinematics for $2.1 \mathrm{GeV}$ electrons. Calculations are shown for the EEI, EDAD1, and IA2 models as solid, dashed, and dotted lines, respectively. 